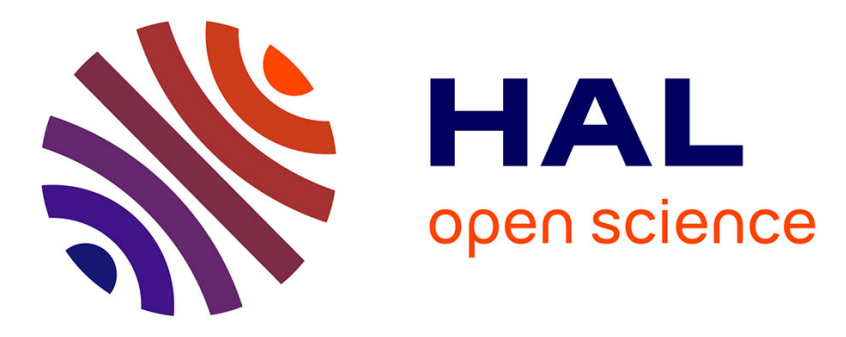

\title{
Conditional toggle mappings: principles and applications
} Santiago Velasco-Forero, Jesus Angulo, Pierre Soille

\section{To cite this version:}

Santiago Velasco-Forero, Jesus Angulo, Pierre Soille. Conditional toggle mappings: principles and applications. Journal of Mathematical Imaging and Vision, 2013, pp.1-12. 10.1007/s10851-013-04294. hal-00797378

\section{HAL Id: hal-00797378 https://hal.science/hal-00797378}

Submitted on 6 Mar 2013

HAL is a multi-disciplinary open access archive for the deposit and dissemination of scientific research documents, whether they are published or not. The documents may come from teaching and research institutions in France or abroad, or from public or private research centers.
L'archive ouverte pluridisciplinaire HAL, est destinée au dépôt et à la diffusion de documents scientifiques de niveau recherche, publiés ou non, émanant des établissements d'enseignement et de recherche français ou étrangers, des laboratoires publics ou privés. 


\title{
Conditional toggle mappings: principles and applications
}

\author{
Santiago Velasco-Forero Jesús Angulo Pierre Soille \\ velasco@cmm.ensmp.fr angulo@cmm.ensmp.fr pierre.soille@jrc.ec.europa.eu
}

February 27, 2013

\begin{abstract}
We study a class of mathematical morphology filters to operate conditionally according to a set of pixels marked by a binary mask. The main contribution of this paper is to provide a general framework for several applications including edge enhancement and image denoising, when it is affected by salt-and-pepper noise. We achieve this goal by revisiting shock filters based on erosions and dilations and extending their definition to take into account the prior definition of a mask of pixels that should not be altered. New definitions for conditional erosions and dilations leading to the concept of conditional toggle mapping. We also investigate algebraic properties as well as the convergence of the associate shock filter. Experiments show how the selection of appropriate methods to generate the masks lead to either edge enhancement or salt-and-pepper denoising. A quantitative evaluation of the results demonstrates the effectiveness of the proposed methods. Additionally, we analyse the application of conditional toggle mapping in remote sensing as pre-filtering for hierarchical segmentation.
\end{abstract}

\section{Introduction}

Whether for edge enhancement or salt-and-pepper denoising, effective methodologies should only alter the values of selected image pixels: those lying along the edges in the former case and those corrupted by salt-and-pepper noise in the latter case. However, most available techniques do not satisfy this condition so that undesirable effects are obtained. For example, halo effects are commonly produced by edge enhancement techniques such as shock filters (see example in [27, Fig. 4]) and noise-free structures are not always preserved when mitigating salt-and-pepper noise with median filters since they round the corners of well defined objects such as rectangles (see example in [38, Fig. 2b]). This motivated us to develop a method achieving either edge enhancement or salt-and-pepper denoising while acting only where deemed necessary. We achieve this goal by revisiting shock filters based on erosions and dilations and extending their definition to take into account the prior definition of a mask of pixels that should not be altered.

The basic idea behind shock filters is to perform dilations and erosions in order to create a shock between the influence zones of the image extrema. Dilations operate around maxima 
and erosion around minima. Most of the current shock filters are based either on the original definition of Kramer and Bruckner in terns of minimum/maximum neighbourhood filters [22] or on modifications of Osher and Rudin formulation in terms of PDEs [29]. Shock filters offer a number of advantages: (i) they create strong discontinuities at image edges, and within a region the filtered signal becomes flat; (ii) they satisfy a maximum-minimum principle so that the range of the filtered image remains within the range of the original image; (iii) they do not increase the L1 norms of the derivatives of a signal (total variation); and (iv) they possess inherent stability properties [52]. Moreover, in contrast to frequency domain methods based on Fourier or wavelet transform, over- and undershoots cannot appear [24]. This makes shock filters attractive for a number of applications where edge sharpening and a piecewise constant segmentation is desired. Several interesting modifications of the original schemes have been proposed, for instance: morphological toggle mappings [27, 37], PDE-based enhancing [11, $30,34,7]$ as well as coherence-enhancing shock filters [52] combining the stability properties of shock filters with the possibility of enhancing flow based on the eigenvalues of the secondmoment matrix or structure tensor. All these variants still pursue the original intention of shock filtering, namely edge enhancement. In many practical situations, the intensity of pixels within an object varies slowly across the 'interior' of objects [8]. A good edge enhancement algorithm should alter pixels near the edges and preserve local contrast for interior pixels. However, the image contrast is highly correlated with the gradient magnitude of its edges [8]. Consequently, some shock filters can cause unpleasant ring or halo effects. This drawback was pointed out by different authors $[27,10]$. Another weakness lies in the large number of iterations needed to converge to a stable result. In this paper, we propose shock filters that do not suffer from halo effects and converge more rapidly than the original definition [22]. We achieve this by introducing the notion of conditional toggle mappings. These mappings rely on erosions and dilations similarly to the original contrast toggle mappings [27] but they act solely on a subset of the image definition domain thanks to the definition of an appropriate mask preventing the modification of the pixels belonging to it. This introduces additional flexibility that enables image denoising in addition to edge enhancement, the type of filtering depending on the method used to produce the mask.

This paper is structured as follows. A review on shock filters based on toggle mappings and an analysis of their convergence properties is given in Sec. 2. Section 3 proposes new definitions for conditional erosions and dilations and the concept of conditional toggle mapping is introduced. We also investigate the properties of the proposed conditional operations including the convergence property of the conditional toggle mapping. Section 4 is devoted to experiments. We show that the selection of appropriate methods to generate the masks lead to either edge enhancement or salt-and-pepper denoising. A quantitative evaluation of the results demonstrates the effectiveness of the proposed methods. Additionally, we analyse the application of conditional toggle mapping in remote sensing as pre-filtering for hierarchical segmentation. All experiments are conducted on grey level and colour images originating from standard image data bases as well very high resolution satellite imagery. The paper 
concludes in Sec. 5 with a summary and perspectives.

\section{Review of the toggle mapping operator}

Let us precise the terms and notation to be used in the rest of the paper. Let $\mathbb{E}$ be a subset of the discrete space $\mathbb{Z}^{2}$, considered as the support space of the image, and $\mathbb{F} \subseteq \mathbb{R}^{d}$ be a set of pixels values in dimension $d$, corresponding to the space of values of the image with $d$ channels. A vector-value image is represented by the mapping,

$$
\mathbf{I}:\left\{\begin{array}{lll}
\mathbb{E} & \rightarrow & \mathbb{F} \\
x & \rightarrow & \mathbf{x}
\end{array}\right.
$$

i.e., $\mathbf{I} \in \mathcal{F}(\mathbb{E}, \mathbb{F})$ the set of maps from a pixel $x \in \mathbb{E}$ into a vector value $\mathbf{x} \in \mathbb{F} \subseteq \mathbb{R}^{d}$. The two basic morphological mappings $\mathcal{F}(\mathbb{E}, \mathbb{F}) \rightarrow \mathcal{F}(\mathbb{E}, \mathbb{F})$ are the dilation $(\delta)$ and erosion $(\varepsilon)$ by a flat structuring element $\mathrm{SE}$ and are given respectively by:

$$
\forall x \in \mathbb{E}, \quad \delta_{\mathrm{SE}}(\mathbf{I})(x)=\bigvee_{y \in \operatorname{SE}(x)} \mathbf{I}(y)
$$

and

$$
\forall x \in \mathbb{E}, \quad \varepsilon_{\mathrm{SE}}(\mathbf{I})(x)=\bigwedge_{y \in \operatorname{SE}(x)} \mathbf{I}(y)
$$

where $\operatorname{SE}(x) \in \mathbb{E}$ denote the spatial neighbourhood induced by the structuring element $\mathrm{SE}$ centred at $x$ and $\breve{S E}$ is the structuring element mirrored in the origin. Note that our definitions (2) and (3) differ from the usual ones in [18] by inverting the structuring element SE. These operators require that $\mathbb{F}$ should be a lattice with suitable operators $\bigvee$ and $\Lambda$. In order to make easier the understanding of our approach, we limit ourselves to the case of grey scale images $(d=1)$. In this case, the $\vee$ and $\wedge$ operators correspond to the supremum and infimum respectively. Thus, images are considered as numerical functions $\mathbb{E} \rightarrow \mathbb{F}$ where $\mathbb{F}=\mathbb{R} \bigcup\{+\infty,-\infty\}$ or $\mathbb{Z} \bigcup\{+\infty,-\infty\}$, or more generally $\mathbb{F}$ is a closed subset of $\mathbb{R}$. The first definition of a shock filter may be traced back to 1975 when Kramer and Bruckner [22] have proposed to use non-linear transformation for image edge enhancement using local minimum and maximum neighbourhood filters (i.e., grey level erosion and dilation). This approach was formalised and generalised by Meyer and Serra [27] in 1989 and led to the notion of toggle mappings [36, 27, 37]. Informally, a toggle mapping is obtained by considering a series of image transformations and a toggling criterion that determines for each spatial position which transformation should be selected for the output. A trivial example of a toggle mapping is the threshold operator. The primitive image transformations are the transformations that set the image pixels to 0 and 1 respectively, and the decision rule involves a simple comparison of the pixel values with a threshold value. The toggle contrast mapping is based on the idea of using a dilation process near a local maximum and an erosion process around a local minimum. 
The decision whether a pixel belongs to the influence zone of a maximum or a minimum is made on the basis of the morphological Laplacian $\Delta$ defined in [50] as the difference between the gradients by dilation $\rho^{\delta}$ and erosion $\rho^{\varepsilon}[31]$ :

$$
\Delta_{\mathrm{SE}}(\mathbf{I})(x)=\rho_{\mathrm{SE}}^{\delta}(\mathbf{I})(x)-\rho_{\mathrm{SE}}^{\varepsilon}(\mathbf{I})(x),
$$

where $\rho_{\mathrm{SE}}^{\delta}(\mathbf{I})(x)=\left(\delta_{\mathrm{SE}}(\mathbf{I})(x)-\mathbf{I}(x)\right)$ and $\rho_{\mathrm{SE}}^{\varepsilon}(\mathbf{I})(x)=\left(\mathbf{I}(x)-\varepsilon_{\mathrm{SE}}(\mathbf{I})(x)\right)$. If the Laplacian is negative, then the pixel is considered to be in the influence zone of a maximum, while it is regarded to belong to the influence zone of a minimum if the Laplacian is positive. With this framework, we adopt the three-state toggle contrast mapping proposed in [34] and based on the erosion, dilation, and identity transformations:

Definition 1 [34] Given an image I the toggle contrast mapping is defined as follows:

$$
\forall x \in \mathbb{E}, \quad \tau_{\mathrm{SE}}(\mathbf{I})(x)= \begin{cases}\varepsilon_{\mathrm{SE}}(\mathbf{I})(x) & \text { if } \Delta_{\mathrm{SE}}(\mathbf{I})(x)>0, \\ \delta_{\mathrm{SE}}(\mathbf{I})(x) & \text { if } \Delta_{\mathrm{SE}}(\mathbf{I})(x)<0, \\ \mathbf{I}(x) & \text { otherwise. }\end{cases}
$$

Note that the original shock filter proposed of Kramer \& Bruckner [22] corresponds to a two-state toggle contrast mapping where the identity transformation is not considered and the strict inequality $<$ in $(4)$ is replaced by $\leq$, see also [27]. The three-state toggle contrast mapping is more robust than the two-state one because it is self-dual and preserve the original signal in a single-slope signal, i.e., signals such that $\forall x: \rho_{\mathrm{SE}}^{\varepsilon}(\mathbf{I}(x))=\rho_{\mathrm{SE}}^{\delta}(\mathbf{I}(x))$. The original method of Kramer and Bruckner was formulated in a fully discrete way. The term shock filtering was introduced later by Osher and Rudin [30]. Shock filtering constitutes an example of a PDE that is difficult to analyse in the continuous setting, while for a 1-D space discretization it has been shown in [53] that this process is well-posed and satisfies a maximum-minimum principle. An analytic solution of the corresponding dynamical system was even found [53]. Different modifications have been proposed in order to improve the performance of shock filters. Alvarez and Mazorra [1] replaced the Laplacian as edge detector by $K_{\sigma} * \Delta(\mathbf{I})$, where $K_{\sigma}$ is a Gaussian with standard deviation $\sigma, \Delta \mathbf{I}$ is the gradient of $\mathbf{I}$, and * denotes convolution. By iterating toggle contrast mappings, a sharp discontinuity (shock) at the borderline between two influence zones is produced. Within each zone, a constant segment is created. Iterations are usually performed until idempotence ${ }^{1}$, i.e.,

$$
\forall x \in \mathbb{E}, \quad \tau_{\mathrm{SE}}^{\infty}(\mathbf{I})(x)=\lim _{m \rightarrow \infty} \tau_{\mathrm{SE}}^{m}(\mathbf{I})(x)
$$

where $\tau_{\mathrm{SE}}^{m}(\mathbf{I})=\tau_{\mathrm{SE}}\left(\tau_{\mathrm{SE}}^{m-1}(\mathbf{I})\right), \tau_{\mathrm{SE}}^{0}(\mathbf{I})(x)=\mathbf{I}(x)$ and $\tau_{\mathrm{SE}}(\mathbf{I})(x)$ is given by $(4)$. The iterations of toggle contrast converge to a fixed point [22] reached after a finite number of iterations. In [16] are defined self-dual operators based on the morphological centre and, more generally,

\footnotetext{
${ }^{1}$ An operator $\phi: \mathbb{E} \rightarrow \mathbb{F}$ is idempotent if $\forall x \in \mathbb{E}, \phi^{2}(x)=\phi(\phi(x))=\phi(x)$.
} 


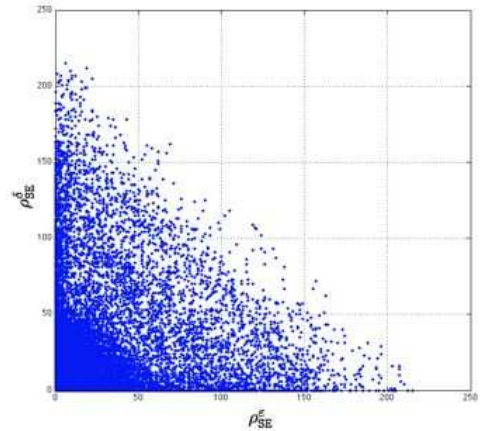

(a) $\rho_{\mathrm{SE}}^{\varepsilon}(\mathbf{I})$ vs $\rho_{\mathrm{SE}}^{\delta}(\mathbf{I})$

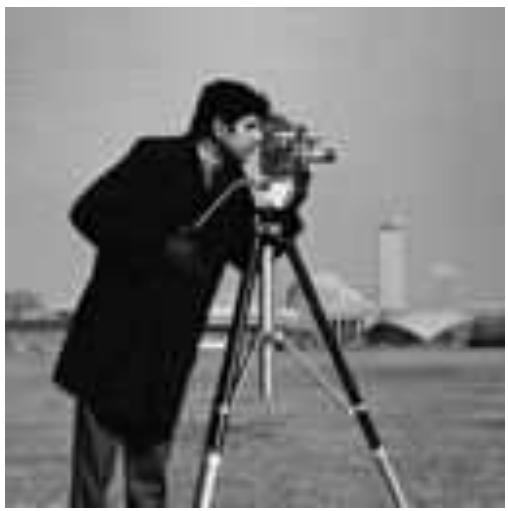

(d) $\mathbf{I}$

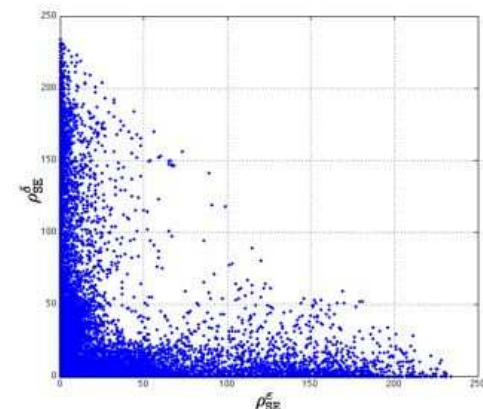

(b) $\rho_{\mathrm{SE}}^{\varepsilon}\left(\tau_{\mathrm{SE}}(\mathbf{I})\right)$ vs $\rho_{\mathrm{SE}}^{\delta}\left(\tau_{\mathrm{SE}}(\mathbf{I})\right)$

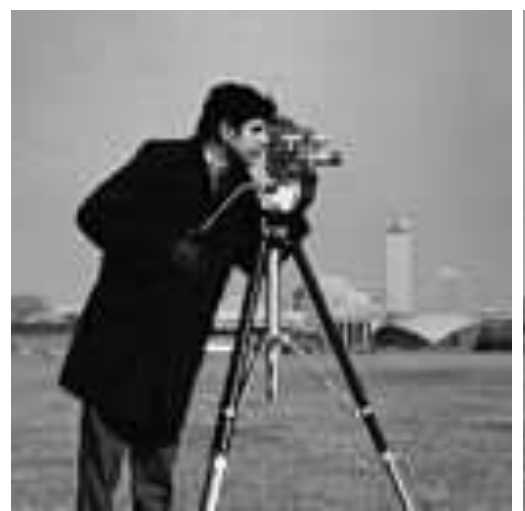

(e) $\tau_{\mathrm{SE}}(\mathbf{I})$

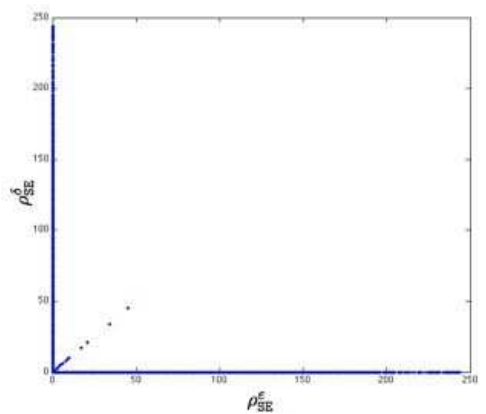

(c) $\rho_{\mathrm{SE}}^{\varepsilon}\left(\tau_{\mathrm{SE}}^{\infty}(\mathbf{I})\right)$ vs $\rho_{\mathrm{SE}}^{\delta}\left(\tau_{\mathrm{SE}}^{\infty}(\mathbf{I})\right)$

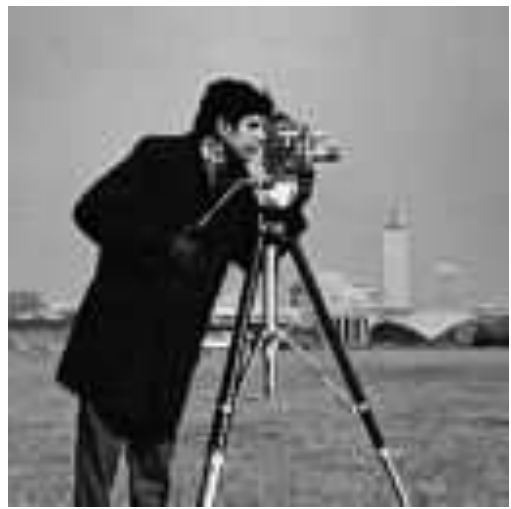

(f) $\tau_{\mathrm{SE}}^{\infty}(\mathbf{I})$

Figure 1: (a)-(c) Bi-dimensional representation of the evolution in the classical shock filter (4) for the Cameraman grey-scale image shown in (d). (e) One iteration of the shock filter in (4) (f) Image obtained after iterating (4) until stability.

self-dual filters. Basically, it states that every increasing, self-dual operator can be modified in such a way that when iterated on any given image, it leads to a pixel-wise monotone sequence (i.e., strictly increasing or decreasing in each pixel). This implies a convergence to a limit operator, thus avoiding oscillation problems which typically hold for non-convergent filters such as median filtering. The convergence is obtained when all the pixels are fixed points, as shown in Fig. 1. To illustrate the evolution until convergence, Figs. 1(a-b) show the first and second iteration of (5) in a bi-dimensional representation of image value gradient by erosion and dilation. When convergence is reached, Fig. 1(c), all the pixels are fixed points according to the criterion (4). Note the presence of pixels in the line $\rho_{\mathrm{SE}}^{\varepsilon}(\mathbf{I})(x)=\rho_{\mathrm{SE}}^{\delta}(\mathbf{I})(x)$ revealing that some pixels have identical values for their gradients by erosion and dilation. Shock filters following (5) have two main drawbacks. First, they suffer from the halo effect $[27,11]$ due to the tie case in the definition. In addition, they require a large number of iterations to converge. For example, in the case of the Cameraman image, 96 iterations were 
needed to reach the stable result shown in Fig. 1(f). These two drawback motived us to define the conditional toggle mapping introduced in the next section.

\section{Conditional toggle mapping}

New definitions for the notion of conditional dilation and erosion are introduced in Sec. 3.1 while studying their properties. These definitions are used as a basis for the conditional toggle mapping proposed in Sec. 3.2. Implementation details are given in Sec. 3.3. Note that the term conditional, applied to our discrete operators, is related to the dependency of the results to a given mask. This term is used as reference of the role played by the mask and should not be confused with the one in conditional geodesic dilation, where a conditional dilation is defined (in the binary case) as the intersection of the mask image with a dilation of the marker image, see for instance [35, Sec. 4.4].

\subsection{Conditional dilation/erosion}

Let $\mathbf{M}$ be the characteristic function of a mask, i.e., $\mathbf{M} \in \mathcal{F}(\mathbb{E},\{0,1\})$ maps each pixel $x \in \mathbb{E}$ into $\{0,1\}$. Our approach is based on a neighbourhood associated to a structuring element SE and to the mask $\mathbf{M}$, denoted by $N$ and defined as follows:

$$
\forall x \in \mathbb{E}, \quad N_{(\mathrm{SE}, \mathbf{M})}(x)=\{y \in \mathrm{SE}(x) \text { and } \mathbf{M}(y)=1\} .
$$

That allows us to define the conditional version of the dilation as the following morphological mapping $\mathcal{F}(\mathbb{E}, \mathbb{F}) \rightarrow \mathcal{F}(\mathbb{E}, \mathbb{F})$.

Definition $\mathbf{2}$ The conditional dilation of an image $\mathbf{I}$ with respect to $\mathbf{M}$ is defined by

$$
\forall x \in \mathbb{E}, \quad \delta_{\mathrm{SE}}(\mathbf{I}, \mathbf{M})(x)= \begin{cases}\underset{y \in N_{(\mathrm{SE}, \mathbf{M})}(x)}{\bigvee} \mathbf{I}(y) & \text { if } \quad x \notin \mathbf{M} \text { and } N_{(\mathrm{SE}, \mathbf{M})}(x) \neq \emptyset, \\ \mathbf{I}(x) & \text { otherwise. }\end{cases}
$$

and similarly for the erosion,

Definition 3 The conditional erosion of an image $\mathbf{I}$ with respect to a binary mask $\mathbf{M}$ is defined by the following expression:

$$
\forall x \in \mathbb{E}, \quad \varepsilon_{\mathrm{SE}}(\mathbf{I}, \mathbf{M})(x)= \begin{cases}\bigwedge_{y \in N_{(\widetilde{S E}, \mathbf{M})}(x)} \mathbf{I}(y) & \text { if } \quad x \notin \mathbf{M} \text { and } N_{(\check{\mathrm{SE}, \mathbf{M})}}(x) \neq \emptyset, \\ \mathbf{I}(x) & \text { otherwise. }\end{cases}
$$

A similar idea of conditional morphology was presented in [20] in the case of binary images. Expression (6) and (7) are equivalent to the ones introduced in [20] for binary images but differs in grey-scale images due to the "otherwise" case. The motivation of this idea is that 


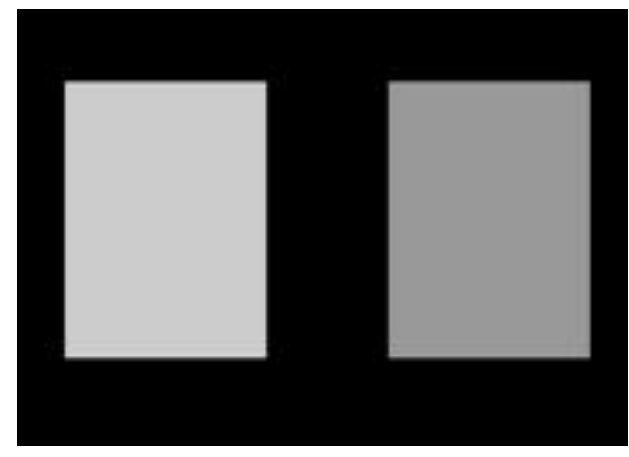

(a) $\mathbf{I}$

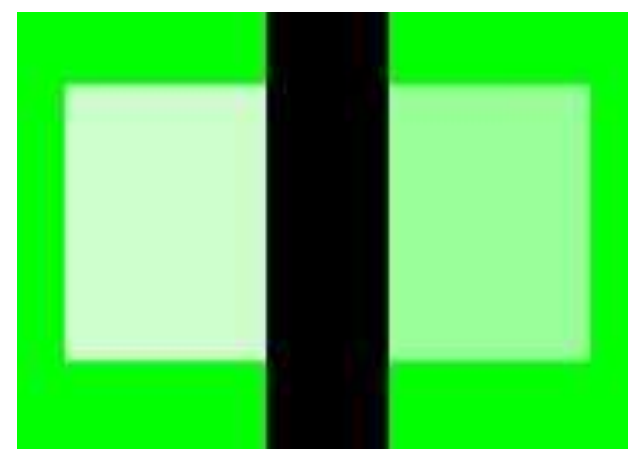

(c) $\mathbf{M}$

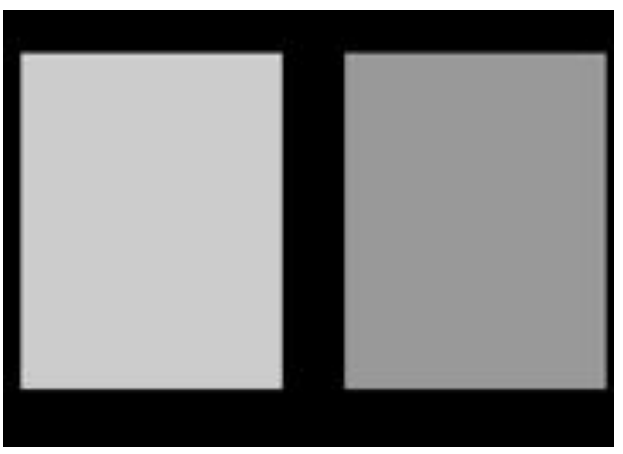

(b) $\delta_{\mathrm{SE}}(\mathbf{I})$

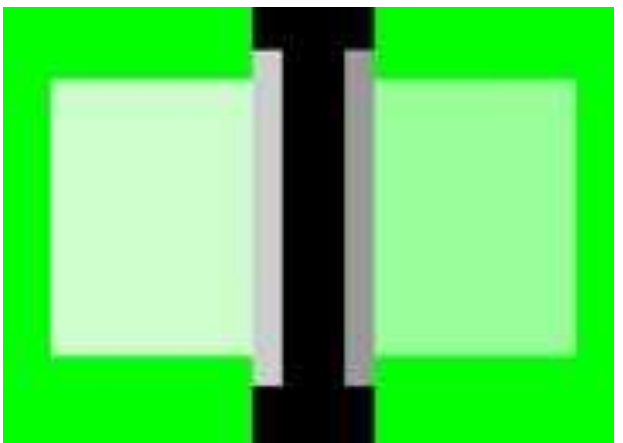

(d) $\delta_{\mathrm{SE}}(\mathbf{I}, \mathbf{M})$

Figure 2: Conditional vs standard operators. I is a grey scale image of $175 \times 245$ pixels (a), $\mathrm{SE}$ is a square of side 25. Pixels of the mask are displayed in green (c). Note that objects in the mask are neither dilated nor eroded for the conditional operators.

pixels in the mask are considered as sources in the morphological operation, and they are invariants (see Fig. 2 for an illustrative example). Algebraic properties of morphological operators have been studied extensively in $[15,16,32,25]$. One of the main ingredients of the theory of the algebraic framework for morphological operators is the definition of an adjunction which forms a Galois connection [18].

Definition $4[9,15]$ Let $\mathcal{L}_{1}$ and $\mathcal{L}_{2}$ be two complete lattices, $\varepsilon$ an operator $\mathcal{L}_{1} \rightarrow \mathcal{L}_{2}$, and $\delta$ an operator $\mathcal{L}_{2} \rightarrow \mathcal{L}_{1}$. The pair $(\varepsilon, \delta)$ is called an adjunction if $\delta(\mathbf{I}) \leq_{\mathcal{L}_{1}} \mathbf{J} \Longleftrightarrow \mathbf{I} \leq \mathcal{L}_{2} \varepsilon(\mathbf{J})$.

We enunciate the equivalence between adjunction and the composition of morphological operators. It can be found as Theorem O.3.6 in [9] or Proposition 2.6 in [18].

Proposition $1[15](\varepsilon, \delta)$ is an adjunction $\Longleftrightarrow \delta$ and $\varepsilon$ are both increasing and $\delta(\varepsilon(\mathbf{J})) \leq \mathcal{L}_{1}$ $\mathbf{J} \quad \forall \mathbf{J} \in \mathcal{L}_{1}$ and $\mathbf{I} \leq_{\mathcal{L}_{2}} \varepsilon(\delta(\mathbf{I})) \quad \forall \mathbf{I} \in \mathcal{L}_{2}$.

We can now note that the pair $\left(\varepsilon_{\mathrm{SE}}(\cdot, \mathbf{M}), \delta_{\mathrm{SE}}(\cdot, \mathbf{M})\right)$ is not an adjunction as it is illustrated in Fig. 3. However, we can calculate the algebraic adjunction of the conditional dilation. 


\begin{tabular}{|c|c|c|c|c|}
\hline$k$ & $k$ & $k$ & $k$ & $k$ \\
\hline$k$ & $k$ & $k$ & $k$ & $k$ \\
\hline$k$ & $k$ & $m$ & $k$ & $k$ \\
\hline$k$ & $k$ & $k$ & $k$ & $k$ \\
\hline$k$ & $k$ & $k$ & $k$ & $k$ \\
\hline
\end{tabular}

(a) $\mathbf{I}$

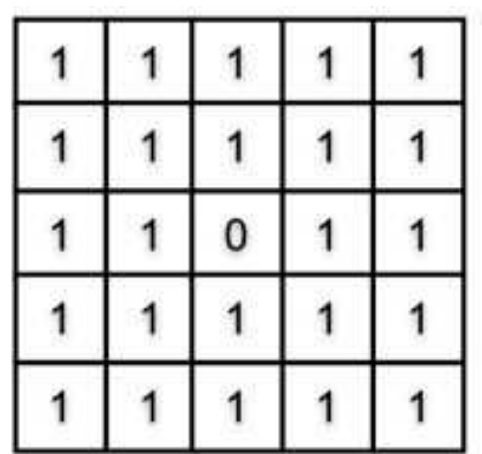

(b) $\mathbf{M}$

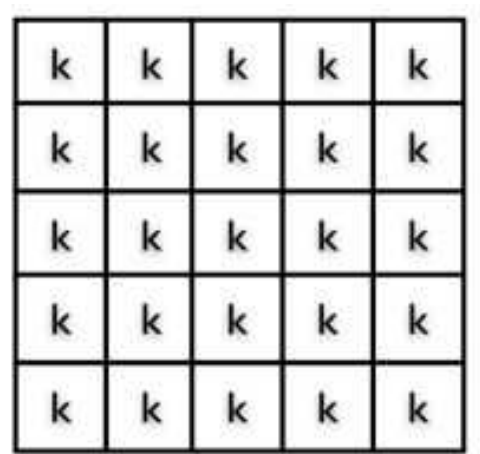

(c) $\delta_{\mathrm{SE}}\left(\varepsilon_{\mathrm{SE}}(\mathbf{I}, \mathbf{M}), \mathbf{M}\right)$

Figure 3: Example showing that the pair conditional operators are not an adjunction in algebraic sense. If $k \neq m, \delta_{\mathrm{SE}}\left(\varepsilon_{\mathrm{SE}}(\mathbf{I}, \mathbf{M}), \mathbf{M}\right)=\varepsilon_{\mathrm{SE}}\left(\delta_{\mathrm{SE}}(\mathbf{I}, \mathbf{M}), \mathbf{M}\right)$ but not equal to $\mathbf{I}$, so by Proposition $1,\left(\varepsilon_{\mathrm{SE}}(\cdot, \mathbf{M}), \delta_{\mathrm{SE}}(\cdot, \mathbf{M})\right)$ is not an adjunction. In the example, $\mathrm{SE}$ is a square of side three pixels (8-connectivity).

It is important because it produces a link in a unique way between morphological operators and idempotent filtering, achieved by composition of dilation/erosion (opening/closing). Additionally, it guarantees that the composition reduces the information content. This kind of analysis has been carried out in morphological operators applied to images [18], graphs [17], pyramids [21], and curve evolution [21].

Proposition 2 Let $\tilde{\varepsilon}_{\mathrm{SE}}(\mathbf{I}, \mathbf{M})$ be defined by:

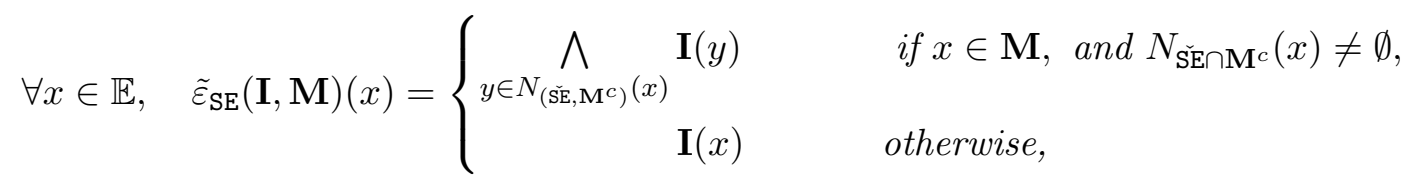

then the pair $\left(\tilde{\varepsilon}_{\mathrm{SE}}(\mathbf{I}, \mathbf{M}), \delta_{\mathrm{SE}}(\mathbf{I}, \mathbf{M})\right)$ is an adjunction.

Proof. For every $\mathrm{SE} \in \mathbb{E}$ and a given $\mathbf{M} \in \mathcal{F}(\mathbb{E},\{0,1\})$, the pair $\left(\tilde{\varepsilon}_{\mathrm{SE}}(\cdot, \mathbf{M}), \delta_{\mathrm{SE}}(\cdot, \mathbf{M})\right)$ defines an adjunction on $\mathcal{F}(\mathbb{E}, \mathbb{F})$ [Proposition 4.33, [15]]. In other words

$$
\delta_{\mathrm{SE}}(\mathbf{I}, \mathbf{M}) \leq \mathbf{J} \Longleftrightarrow \mathbf{I} \leq \tilde{\varepsilon}_{\mathrm{SE}}(\mathbf{J}, \mathbf{M})
$$

for all $\mathbf{I}, \mathbf{J} \in \mathcal{F}(\mathbb{E}, \mathbb{F})$. Following the suggestion by Roerdink [33] a simple proof is obtained based on the observation that the conditional dilation in equation (6) can be rewritten as a space-adaptive dilation, i.e.,

$$
\forall x \in \mathbb{E}, \delta_{\mathrm{SE}(\cdot, \mathrm{M})}(\mathbf{I})(x)=\bigwedge_{y \in N(x)} \mathbf{I}(y)
$$




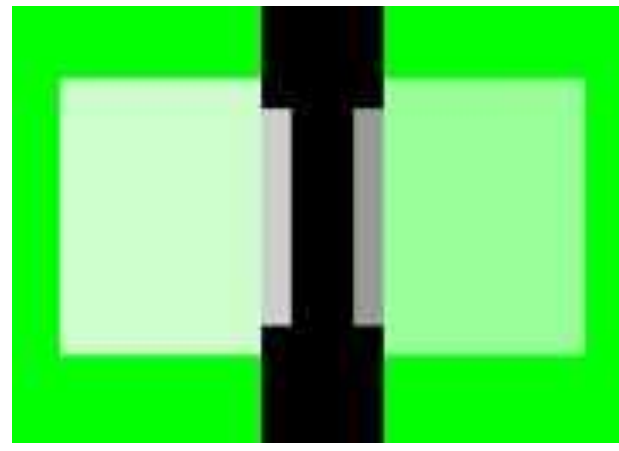

(a) $\varepsilon_{\mathrm{SE}}(\mathbf{I}, \mathbf{M})$

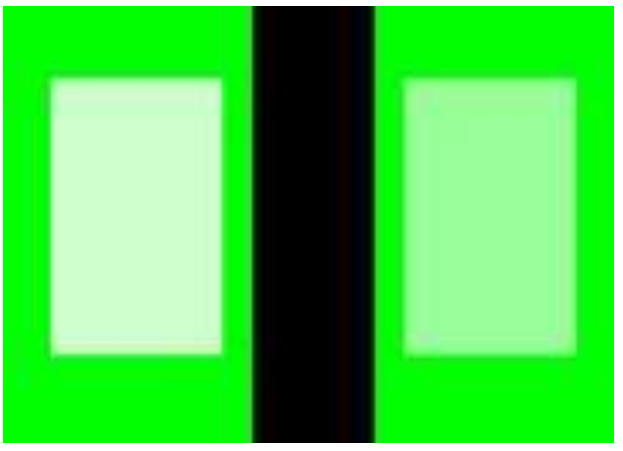

(b) $\tilde{\varepsilon}_{\mathrm{SE}}(\mathbf{I}, \mathbf{M})$

Figure 4: $\left(\tilde{\varepsilon}_{\mathrm{SE}}(\mathbf{I}, \mathbf{M}), \delta_{\mathrm{SE}}(\mathbf{I}, \mathbf{M})\right)$ is an adjunction whereas $\left(\varepsilon_{\mathrm{SE}}(\mathbf{I}, \mathbf{M}), \delta_{\mathrm{SE}}(\mathbf{I}, \mathbf{M})\right)$ is not. $\tilde{\varepsilon}_{\mathbf{S E}}(\mathbf{I}, \mathbf{M})$ only operates on the mask $\mathbf{M}$ in contrast with $\varepsilon_{\mathbf{S E}}(\mathbf{I}, \mathbf{M})$ that performs outside of $\mathbf{M}$.

where the spatial-varying structuring element $N(x)$ is defined as (using the set notation for the mask $\mathbf{M})$ :

$$
\forall x \in \mathbb{E}, N(x)= \begin{cases}\mathrm{SE}(x) \cap \mathbf{M} & \text { if } x \notin \mathbf{M} \text { and } \mathrm{SE}(x) \cap \mathbf{M} \neq \emptyset \\ \{x\} & \text { otherwise. }\end{cases}
$$

Then the space-varying adjoint erosion [32], written $\tilde{\varepsilon}_{\mathrm{SE}(; \mathrm{M})}$, is defined as

$$
\forall x \in \mathbb{E}, \tilde{\varepsilon}_{\mathrm{SE}(\cdot, \mathbf{M})}(x)=\bigwedge_{y \in \tilde{N}(x)} \mathbf{I}(y)
$$

where $\check{N}(x)$ is the reflected structuring element defined by $y \in \check{N}(x) \Longleftrightarrow x \in N(y)$. Accordingly, it can be seen that

$$
\forall x \in \mathbb{E}, \check{N}(x)= \begin{cases}\check{\mathrm{SE}}(x) \cap \mathbf{M}^{c} & \text { if } x \notin \mathbf{M}^{c} \text { and } \check{\mathrm{SE}}(x) \cap \mathbf{M}^{c} \neq \emptyset \\ \{x\} & \text { otherwise. }\end{cases}
$$

which corresponds to the neighbourhood in (8). - The adjoint operator only changes pixels on the mask $\mathbf{M}$ as it is illustrated in Fig. 4 for the same example of Fig. 2. However, in the practical applications considered in this paper the adjoint operator $\tilde{\varepsilon}_{\mathrm{SE}}(\cdot, \cdot)$ does not have any interest. Finally, we present a list of properties for the conditional morphological operators defined in (6) and (7). Let $\varepsilon_{\mathrm{SE}}(\cdot, \cdot), \delta_{\mathrm{SE}}(\cdot, \cdot)$ be the pair of conditional operators. Let define the composition of conditional operation as follows, $\phi_{\mathrm{SE}}(\mathbf{I}, \mathbf{M})=\varepsilon_{\mathrm{SE}}\left(\delta_{\mathrm{SE}}(\mathbf{I}, \mathbf{M}), \mathbf{M}\right)$ and 
$\gamma_{\mathrm{SE}}(\mathbf{I}, \mathbf{M})=\delta_{\mathrm{SE}}\left(\varepsilon_{\mathrm{SE}}(\mathbf{I}, \mathbf{M}), \mathbf{M}\right)$, Accordingly, the following properties hold:

(a) $\quad \varepsilon_{\mathrm{SE}}(\mathbf{I}, \mathbf{M})=\varepsilon_{\mathrm{SE}}\left(\varepsilon_{\mathrm{SE}}(\mathbf{I}, \mathbf{M}), \mathbf{M}\right),($ idempotence);

(b) $\quad \delta_{\mathrm{SE}}(\mathbf{I}, \mathbf{M})=\delta_{\mathrm{SE}}\left(\delta_{\mathrm{SE}}(\mathbf{I}, \mathbf{M}), \mathbf{M}\right)$, (idempotence);

(c) $\bigwedge_{i=1}^{m} \varepsilon_{\mathrm{SE}}\left(\mathbf{I}_{i}, \mathbf{M}\right)=\varepsilon_{\mathrm{SE}}\left(\bigwedge_{i=1}^{m} \mathbf{I}_{i}, \mathbf{M}\right)$, (distributivity);

(d) $\bigvee_{i=1}^{m} \delta_{\mathrm{SE}}\left(\mathbf{I}_{i}, \mathbf{M}\right)=\delta_{\mathrm{SE}}\left(\bigvee_{i=1}^{m} \mathbf{I}_{i}, \mathbf{M}\right)$, (distributivity);

(e) $\mathbf{I} \leq \mathbf{J} \Rightarrow \varepsilon_{\mathrm{SE}}(\mathbf{I}, \mathbf{M}) \leq \varepsilon_{\mathrm{SE}}(\mathbf{J}, \mathbf{M})$, (increasingness);

(f) $\quad \mathbf{I} \leq \mathbf{J} \Rightarrow \delta_{\mathrm{SE}}(\mathbf{I}, \mathbf{M}) \leq \delta_{\mathrm{SE}}(\mathbf{J}, \mathbf{M})$, (increasingness);

(g) $\quad \delta_{\mathrm{SE}}(\cdot, \cdot)$ and $\varepsilon_{\mathrm{SE}}(\cdot, \cdot)$ are morphological filters;

(h) $\quad \gamma_{\mathrm{SE}}(\mathbf{I}, \mathbf{M})=\delta_{\mathrm{SE}}(\mathbf{I}, \mathbf{M})$;

(i) $\quad \phi_{\mathrm{SE}}(\mathbf{I}, \mathbf{M})=\varepsilon_{\mathrm{SE}}(\mathbf{I}, \mathbf{M})$;

$(j) \quad \varepsilon_{\mathrm{SE}}(\mathbf{I}, \mathbf{M}) \leq \delta_{\mathrm{SE}}(\mathbf{I}, \mathbf{M})$;

(k) $\quad \varepsilon_{\mathrm{SE}}(\mathbf{I}, \mathbf{M})=\mathbf{t}_{\max }-\left(\delta_{\mathrm{SE}}\left(\mathbf{t}_{\max }-\mathbf{I}, \mathbf{M}\right)\right)$, (duality).

where, $\mathbf{t}_{\max }=\max (\mathbf{I})$. Note that properties (a) and (b) are unusual, but they illustrate the mask effect included in $\mathbf{M}$. Additionally, note the fact that $\delta_{\mathrm{SE}}(\cdot, \cdot)$ (respectively $\varepsilon_{\mathrm{SE}}(\cdot, \cdot)$ ) is not extensive ${ }^{2}$ (anti-extensive), even if the SE contains the origin. The demonstration of these properties is straightforward from the definition of conditional operators and therefore omitted.

\subsection{Conditional toggle mapping}

As discussed before, we keep the definition $\varepsilon_{\mathrm{SE}}(\cdot, \cdot)$ for the definition of the conditional toggle criterion and define the conditional Laplacian as follows:

$$
\Delta_{\mathrm{SE}}(\mathbf{I}, \mathbf{M})=\left(\delta_{\mathrm{SE}}(\mathbf{I}, \mathbf{M})-\mathbf{I}\right)-\left(\mathbf{I}-\varepsilon_{\mathrm{SE}}(\mathbf{I}, \mathbf{M})\right) .
$$

Definition $\mathbf{5}$ The conditional toggle criterion based on $\mathbf{M}$ is defined as follows:

$$
\forall x \in \mathbb{E}, \quad \tau_{\mathrm{SE}}(\mathbf{I}, \mathbf{M})= \begin{cases}\varepsilon_{\mathrm{SE}}(\mathbf{I}, \mathbf{M}) & \text { if } \quad \Delta_{\mathrm{SE}}(\mathbf{I}, \mathbf{M})>0 \\ \delta_{\mathrm{SE}}(\mathbf{I}, \mathbf{M}) & \text { if } \quad \Delta_{\mathrm{SE}}(\mathbf{I}, \mathbf{M})<0 \\ \mathbf{I} & \text { otherwise }\end{cases}
$$

The motivation behind definition (9) is that the mask $\mathbf{M}$ plays the role of a seed indicator, where the pixel values spread through the image $\mathbf{I}$ according to the toggling criterion. Similarly to non-conditional toggle mapping, conditional toggle mapping should be applied iteratively. In this point, the detector matrix $\mathbf{M}$ have to spread their values through the image.

\footnotetext{
${ }^{2}$ An operator $\phi: \mathbb{E} \rightarrow \mathbb{F}$ is extensive (resp. anti-extensive) if $\forall x \in \mathbb{E}, \phi(x) \geq x$ (resp. $\leq$ ).
} 
Thus, we define a mapping $\tilde{\tau}_{\mathrm{SE}}(\cdot, \cdot)$ from and onto the pair image $\mathbf{I}$ and the mask $\mathbf{M}$ by taking (9) on $\mathbf{I}$ and (2) on $\mathbf{M}$, i.e., $\tilde{\tau}_{\mathrm{SE}}$ is a mapping $\mathcal{F}(\mathbb{E}, \mathbb{F}) \times \mathcal{F}(\mathbb{E},\{0,1\}) \rightarrow(\mathcal{F}(\mathbb{E}, \mathbb{F}), \mathcal{F}(\mathbb{E},\{0,1\}))$ such that

$$
\tilde{\tau}_{\mathrm{SE}}(\mathbf{I}, \mathbf{M})=\left(\tau_{\mathrm{SE}}(\mathbf{I}, \mathbf{M}), \delta_{\mathrm{SE}}(\mathbf{M})\right)
$$

Accordingly, the next iteration can be calculated as follows:

$$
\begin{aligned}
\tilde{\tau}_{\mathrm{SE}}^{2}(\mathbf{I}, \mathbf{M}) & =\tilde{\tau}_{\mathrm{SE}}\left(\tilde{\tau}_{\mathrm{SE}}(\mathbf{I}, \mathbf{M})\right) \\
& =\tilde{\tau}_{\mathrm{SE}}\left(\tau_{\mathrm{SE}}(\mathbf{I}, \mathbf{M}), \delta_{\mathrm{SE}}(\mathbf{M})\right) \\
& =\left(\tau_{\mathrm{SE}}\left(\tau_{\mathrm{SE}}(\mathbf{I}, \mathbf{M}), \delta_{\mathrm{SE}}(\mathbf{M})\right), \delta_{\mathrm{SE}}\left(\delta_{\mathrm{SE}}(\mathbf{M})\right)\right) \\
& =\left(\tau_{\mathrm{SE}}\left(\tilde{\tau}_{\mathrm{SE}}(\mathbf{I}, \mathbf{M})\right), \delta_{\mathrm{SE}}^{2}(\mathbf{M})\right)
\end{aligned}
$$

Finally, the conditional toggle mapping is defined by iteration until convergence $\tilde{\tau}_{\mathrm{SE}}^{\infty}$ as follows.

Definition 6 The conditional toggle mapping based on $\mathbf{M}$ is defined by

$$
\forall x \in \mathbb{E}, \quad \tilde{\tau}_{\mathrm{SE}}^{\infty}(\mathbf{I}, \mathbf{M})=\lim _{m \rightarrow \infty} \tilde{\tau}_{\mathrm{SE}}^{m}(\mathbf{I}, \mathbf{M})
$$

where $\tilde{\tau}_{\mathrm{SE}}^{m}(\mathbf{I}, \mathbf{M})=\tau_{\mathrm{SE}}\left(\tilde{\tau}_{\mathrm{SE}}^{m-1}(\mathbf{I}, \mathbf{M}), \delta_{\mathrm{SE}}^{m}(\mathbf{M})\right)$ and $\tilde{\tau}_{\mathrm{SE}}^{0}(\mathbf{I}, \mathbf{M})=(\mathbf{I}, \mathbf{M})$.

We can also prove that the convergence of the conditional toggle mapping depends on the mask $\mathbf{M}$.

Proposition 3 If $m^{\mathbf{D}} \geq \max \left(\mathbf{D}_{\mathrm{SE}}(\mathbf{M})\right)$ then $\tilde{\tau}_{\mathrm{SE}}^{m^{\mathbf{D}}}(\mathbf{I}, \mathbf{M})$ converges to $\tilde{\tau}_{\mathrm{SE}}^{\infty}$, where $\mathbf{D}_{\mathrm{SE}}(\mathbf{M})$ is the distance transform of the binary image $\mathbf{M}$ associated with connectivity induced by the structuring element SE containing the origin.

Proof. Let $\mathbf{M}: \mathbb{Z}^{2} \rightarrow[0,1]$ be a binary image. The distance transform (DT) is the transformation that generates a map $\mathbf{D}$ whose value in each pixel $x$ is the smallest distance from this pixel to $\mathbf{M}$, i.e.,

$$
\forall x \in \mathbb{E}, \quad \mathbf{D}_{\mathrm{SE}}(\mathbf{M})(x)=\min \left\{m \in \mathbb{N} \mid x \in \delta_{\mathrm{SE}}^{m}(\mathbf{M})\right\} .
$$

It is important to note that SE in (12) must contain the origin, otherwise the standard dilation $\delta_{\mathrm{SE}}(\mathbf{M})$ is not extensive and cannot be used to define a distance transformation $\mathbf{D}_{\mathrm{SE}}$. Let $m \geq$ $m^{\mathbf{D}}=\max \left(\mathbf{D}_{\mathrm{SE}}(\mathbf{M})\right)$ so we have $\delta_{\mathrm{SE}}^{m+1}(\mathbf{M})=\delta_{\mathrm{SE}}^{m}(\mathbf{M})=\mathbf{1}$, where $\mathbf{1}$ is the indicator image of $\mathbb{Z}^{2}$. Accordingly, by definition of conditional morphology, $\varepsilon_{\mathrm{SE}}(\mathbf{I}, \mathbf{1})=\delta_{\mathrm{SE}}(\mathbf{I}, \mathbf{1})=\tau_{\mathrm{SE}}(\mathbf{I}, \mathbf{1})=\mathbf{I}$. Therefore, $\tilde{\tau}_{\mathrm{SE}}^{m}(\mathbf{I}, \mathbf{M})=\tilde{\tau}_{\mathrm{SE}}^{m}(\mathbf{I}, \mathbf{1})=\tilde{\tau}_{\mathrm{SE}}^{m+1}(\mathbf{I}, \mathbf{1})=\tilde{\tau}_{\mathrm{SE}}^{m+1}(\mathbf{I}, \mathbf{M})$. Proposition 3 means that expression (11) converges in no more iterations than $m^{\mathbf{D}}=\max \left(\mathbf{D}_{\mathrm{SE}}(\mathbf{M})\right)$, where $\mathbf{D}_{\mathrm{SE}}(\mathbf{M})$ is the distance transform of $\mathbf{M}$ with connectivity induced by the structuring element SE. That point is important because the non-conditional toggle mapping requires a large number of iteration until convergence. 


\subsection{Implementation}

The pseudocode of the elementary conditional toggle mapping procedure called ToggleMaskElem is displayed in Alg. 1. Note that the input image and mask are modified by this procedure (they contain the conditional toggle mapping output and dilated mask respectively). The called procedures Erode (resp. Dilate) have an image and structuring element as inputs and return the corresponding eroded (resp. dilated) image. The procedure Mult returns the pixel-wise multiplication between the two given input image.

The pseudocode of the elementary conditional toggle mapping procedure called ToggleMaskStable is displayed in Alg. 2. This procedure overwrites the input image and it holds the desired output upon completion. Note that the mask image is also modified since it is dilated until the iterations of the elementary toggle mapping converge. The procedure sum returns the sum of the intensity values of the given input images.

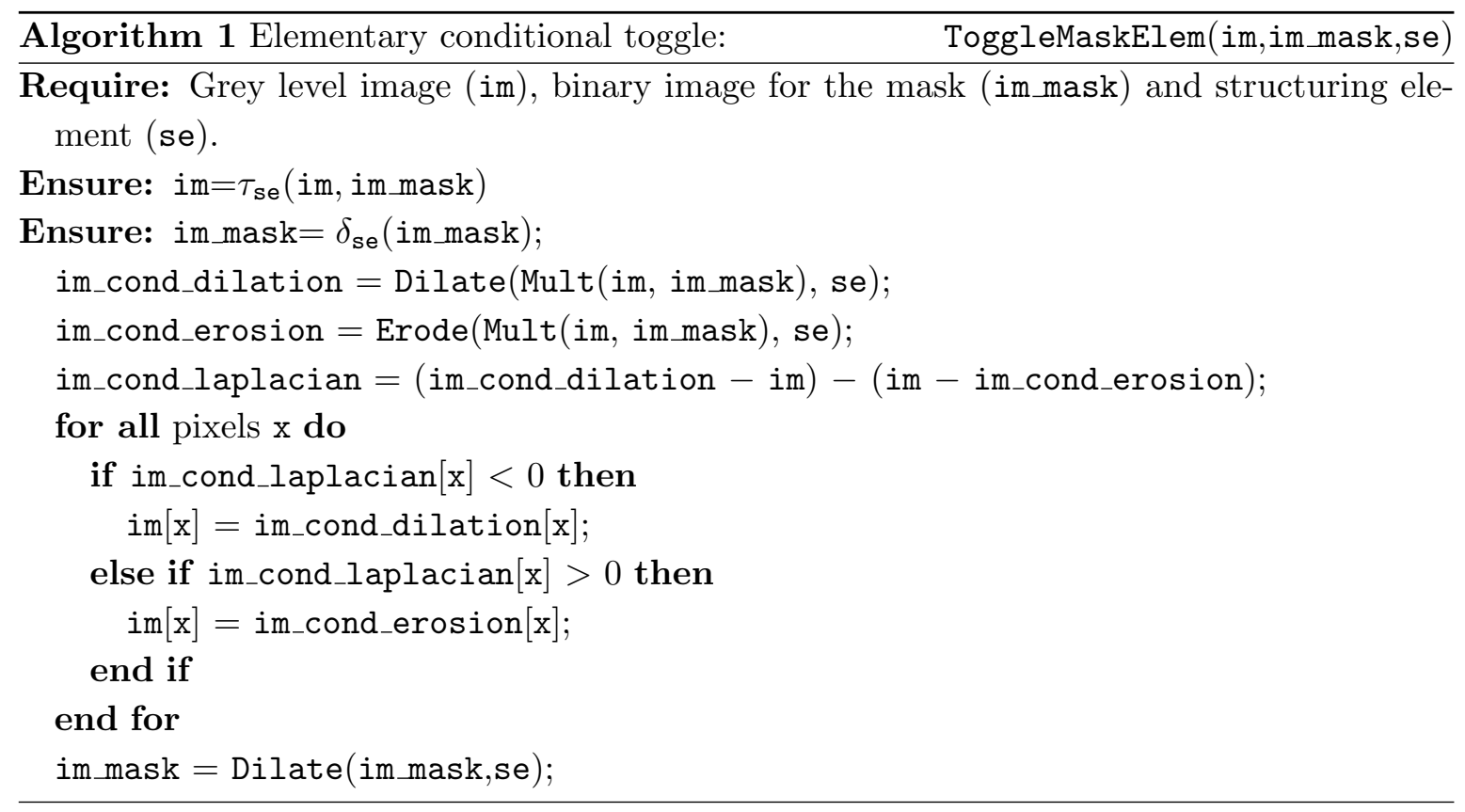

\section{Experiments}

We have developed a conditional shock filtering based on the information contained on a binary mask. A key contribution of this paper is to consider a general formulation where different image processing problem can be considered. In order to test the conditional toggle mapping, we conducted experiments on classical examples of grey and colour images as well as in high resolution remote sensing images. The key question in conditional morphology is how to define the mask $\mathbf{M}$. First, we propose a mask $\mathbf{M}_{E}$ based on local max/minimum pixels and 


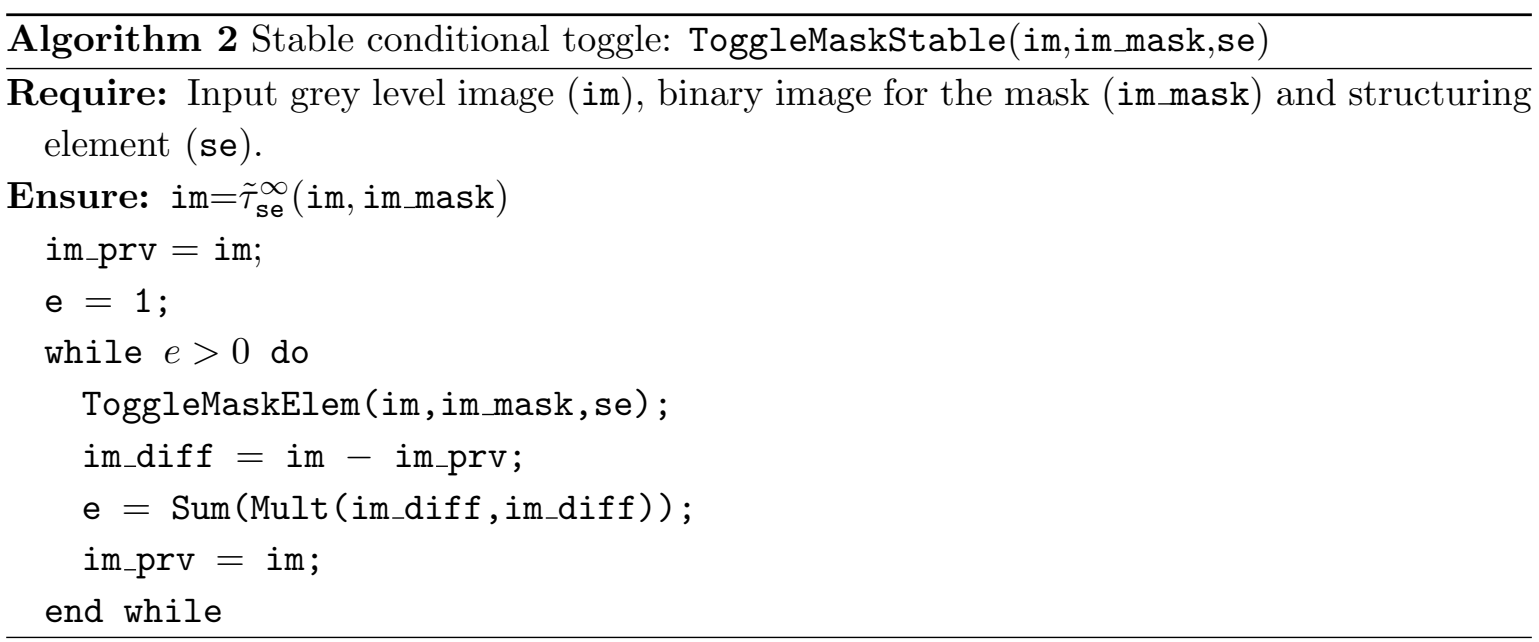

we report the results on edge enhancement on some synthetic signals and images. Second, we proposed a mask $\mathbf{M}_{N}$ based on a simple noise detector and we explore its advantages in the task of salt-and-pepper noise reduction. Third, we analyse the benefits of conditional toggle mapping using $\mathbf{M}_{E}$ as a adequate preprocessing on high-resolution remote sensing images to avoid problematic segmentations.

\subsection{Edge enhancement}

If the main interest is edge enhancement, $\mathbf{M}$ should consist of the local maximum/minimum pixels, because by definition they cannot represent a mixture between the intensity values of two adjacent regions with different intensity levels and therefore should not be modified. This means that the grey value at a local maximum or minimum must not increase or decrease, respectively. For this purpose, we propose to define the mask from the notion of local extrema detailed in [42]:

$$
\forall x \in \mathbb{E}, \mathbf{M}_{E}(x)= \begin{cases}1 & \text { if } \min \left(\rho_{\mathrm{SE}}^{\varepsilon}(x), \rho_{\mathrm{SE}}^{\delta}(x)\right)=0 \\ 0 & \text { otherwise. }\end{cases}
$$

Other possibilities that can be considered are the classical edge detector as it is shown in Fig. 5, the morphological gradient [31] or the inverse of geodesic path distance as in [14]. Figure 5 illustrates different edge detectors and their associated local extrema (pixels considered as local maxima or local minima) according to different definitions. Figure 6 compares the number of iterations until convergence for the classical shock filter given by expression (5) and the proposed one based on conditional mathematical morphology defined by (11) in the case of the Cameraman image of Fig. 1d. We can clearly observe the faster convergence of (11) in comparison with (4) as expected from Prop. 3.

To illustrate the advantages and drawbacks of our approach, we present results on a series of synthetic images. First, let us consider the one dimensional signal examples given in Figs. 


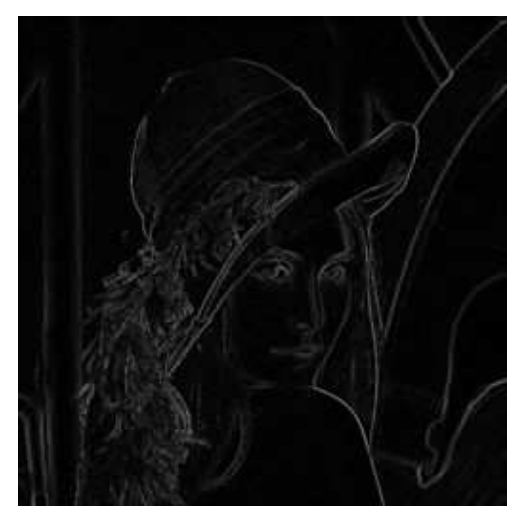

(a) $\min \left\{\rho_{\mathrm{SE}}^{\varepsilon}(\mathbf{I}), \rho_{\mathrm{SE}}^{\delta}(\mathbf{I})\right\}$

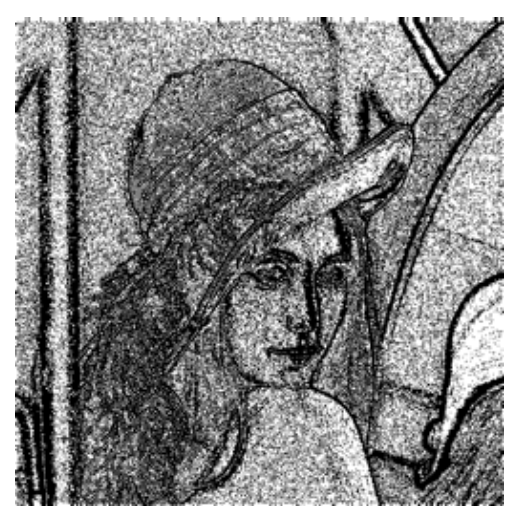

(d) Local extrema of $\min \left\{\rho_{\mathrm{SE}}^{\varepsilon}(\mathbf{I}), \rho_{\mathrm{SE}}^{\delta}(\mathbf{I})\right\}$

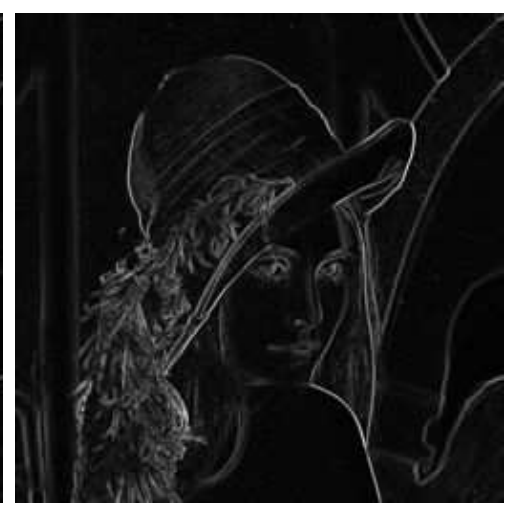

(b) $\frac{\Delta_{\mathrm{SE}}(\mathbf{I})}{2}$

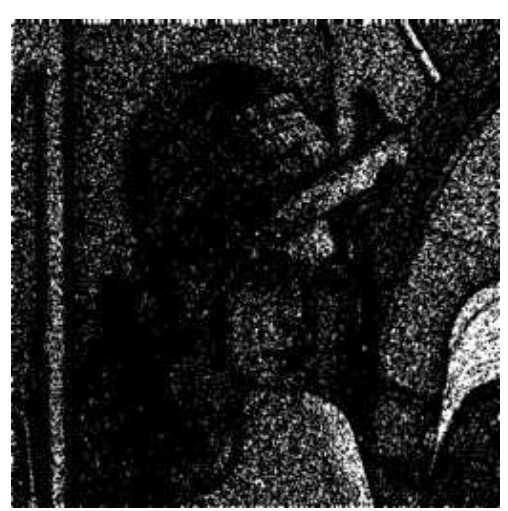

(e) Local extrema of $\frac{\Delta_{\mathrm{SE}}(\mathbf{I})}{2}$

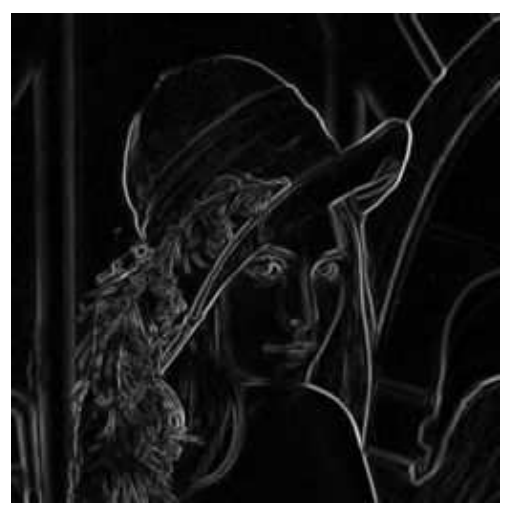

(c) $K_{\sigma} * \Delta \mathbf{I}, \sigma=1$

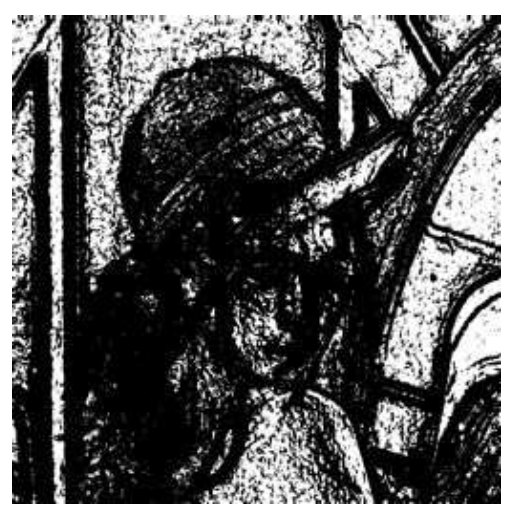

(f) Local extrema of $\left(K_{\sigma} * \Delta \mathbf{I}\right)$

Figure 5: (a-c) Proposed Gradient and other definitions by [22] and [1]. SE is a disk of size 1 and $\sigma=1$. (d-f) Local extrema detector $(<2)$ by using (a-c). 


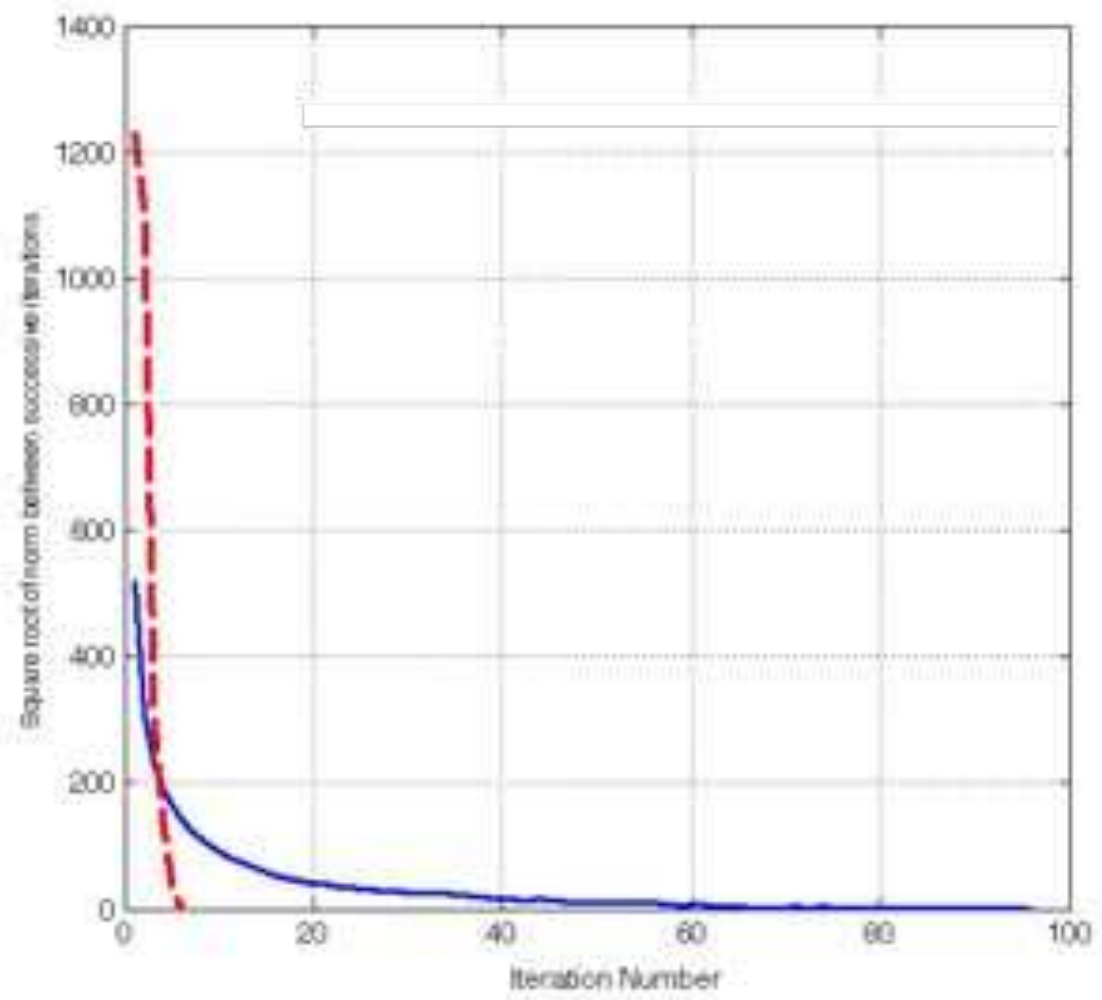

Figure 6: Iteration number versus residual between two consecutive iterations of classical $\left\|\tau_{\mathrm{SE}}^{i+1}(\mathbf{I})-\tau_{\mathrm{SE}}^{i}(\mathbf{I})\right\|$ and conditional $\left\|\tilde{\tau}_{\mathrm{SE}}^{i+1}(\mathbf{I}, \mathbf{M})-\tilde{\tau}_{\mathrm{SE}}^{i}(\mathbf{I}, \mathbf{M})\right\|$ approaches in a grey scale image of size $256 \times 256$ pixels. The proposed conditional toggle mapping converges in nine iterations in comparison with ninety-four in the original one. In terms of processing time, the original toggle mapping takes at least four times than conditional toggle mapping (110 $\mathrm{ms}$ in a 2.4 GHz Intel Core 2 Duo with 4 GB 1067 MHz.) 


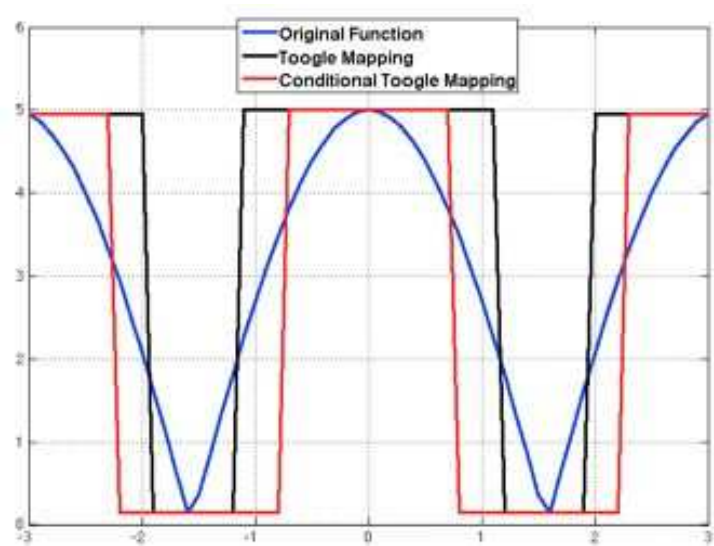

(a) $f(x)=|5 \cos (x)|$

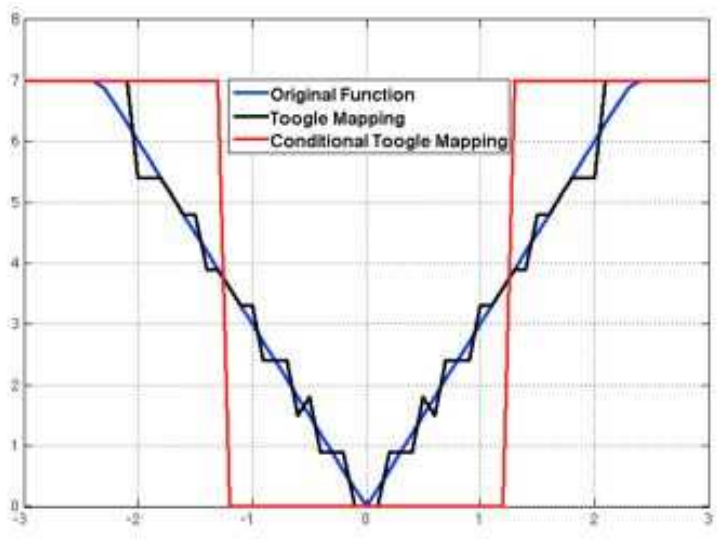

(b) $f(x)=\min (7,3|x|)$

Figure 7: The proposed conditional toggle contrast does not produce halos as classical filters in ramp signals, i.e., oscillate signal in (b). $f(x)=\min (7,3|x|)$ with $x=[-3,3]$, SE is a symmetric window of length 0.5 .

7 (a) and (b) which illustrate the outperformance of the proposed conditional toggle mapping with respect to the traditional shock filter. In the sinusoidal signal, $f(x)=|5 \cos (x)|$, the behaviour of classical and proposed approaches are both accurate. However, classical shock filter fails in the ramp case, $f(x)=\min (7,3|x|)$, as it is illustrated in Fig. 7(b). In this case, proposed conditional transformation operates without including obnoxious halos. At this point, we can see two advantages in contrast with classical toggle mapping: conditional exhibits a faster convergence coupled with robustness to halo effects.

Second, we consider the synthetic case of the convolution of a square with a symmetric Gaussian low pass filter, as it is shown in Fig. 8. Additionally, in the right, a random colour is assigned to each flat zone ${ }^{4}$ of the grey scale image placed in the left. For instance, the original image in Fig. 8(a) and its flat zones in Fig. 8(b). As can be seen, the proposed approach does not include neither ramp nor halo as in the case of classical toggle mapping Fig. 8(e), anisotropic shock filter Fig. 8(i) and complex diffusion Fig. 8(k). However, it is important to note that conditional toggle mapping fails in the correct identification of the corner of the object. This negative point comes from the choice of a classical dilation in the second term of (10). Spatial-adaptive dilation [32] can be considered to avoid this problem, but it is beyond the scope of this paper.

\footnotetext{
${ }^{4}$ Flat zones of $\mathbf{I}$ are the connected component where the intensity does not change.
} 


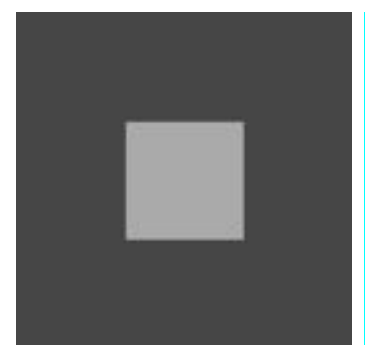

(a) I

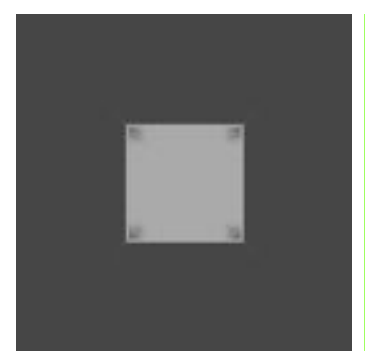

(e) $\tau_{\mathrm{SE}}^{\infty}((\mathrm{c}))$

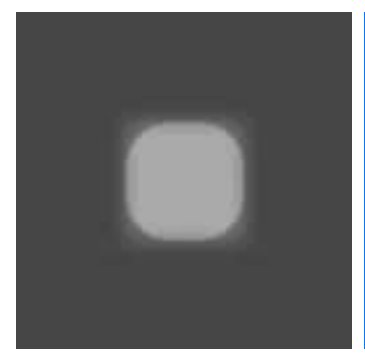

(i) Anisotropic shock filter in [1]

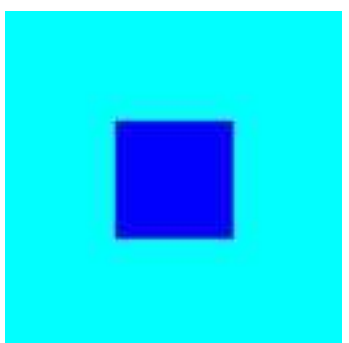

(b) $\mathrm{CC}_{0}(\mathbf{I})$

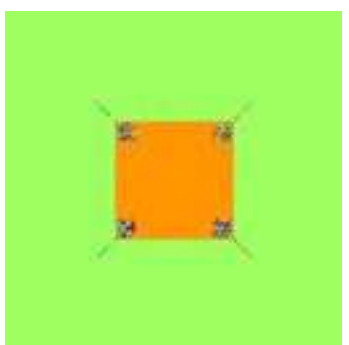

(f) $\mathrm{CC}_{0}((\mathrm{e}))$

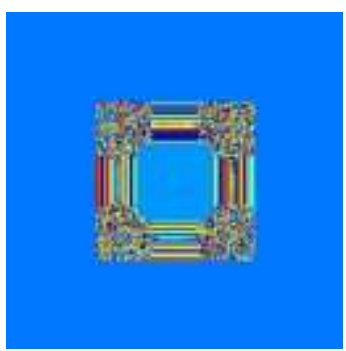

(j) $\mathrm{CC}_{0}((\mathrm{i}))$

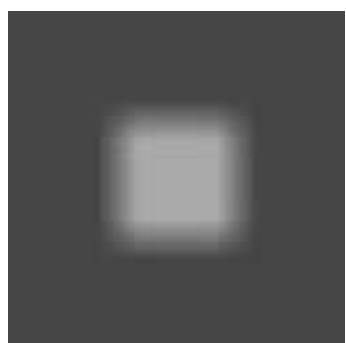

(c) $\mathbf{I} * K_{\sigma}$

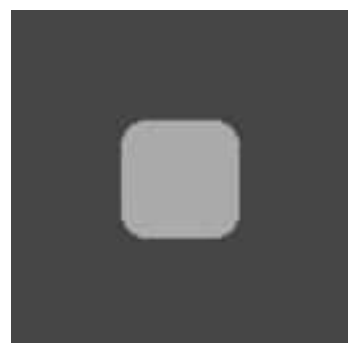

(g) $\tilde{\tau}_{\mathrm{SE}}^{\infty}\left((\mathrm{c}), \mathbf{M}_{E}\right)$

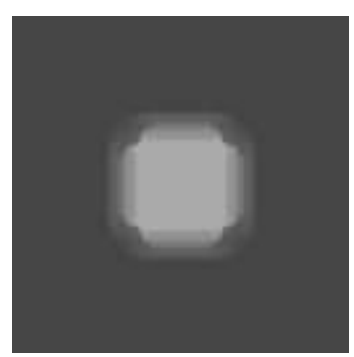

(k) Complex diffusion in $[10]$

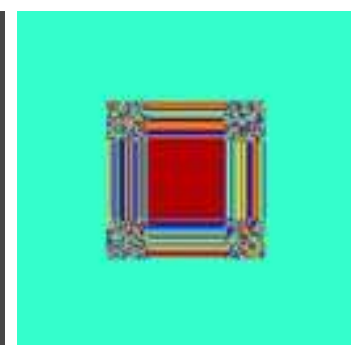

(d) $\mathrm{CC}_{0}\left(\mathbf{I} * K_{\sigma}\right)$

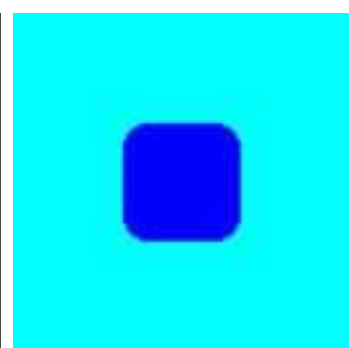

(h) $\mathrm{CC}_{0}((\mathrm{~g}))$

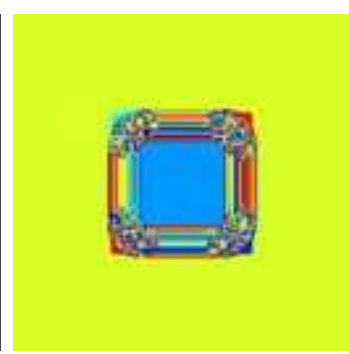

(l) $\mathrm{CC}_{0}((\mathrm{k}))$

Figure 8: Synthetic case: (a) A square of side 71 pixels with grey value equal to 121 is generated on a homogenous background of value 70 and size $200 \times 200$ pixels. (c) is the convolution of (a) with a symmetric Gaussian lowpass filter of size and $\sigma$ equal to 25 . (e) Classical toggle mapping applied on the image (c). Note the halos produced in the corners of the square. (g) Conditional toggle mapping computed on (c). Conditional approach produces sharp edges but it fails in the identification of the square shape of the original image (a). The flat zones (or $\mathrm{CC}_{\alpha}$ with $\alpha=0$ ) of (e) and (g) are illustrated in (f) and (h). 


\subsection{Salt \& pepper noise reduction}

An advantage of the conditional toggle mapping in (11), is that its convergence and mathematical properties are valid for any definition of the binary image $\mathbf{M}$. To illustrate this benefit, we introduce a mask definition for impulse noise removal applications. In this case, $\mathbf{M}$ should have the pixels that are not corrupted by the impulse noise. We propose a simple noise detector as follows,

$$
\forall x \in \mathbb{E}, \quad \mathbf{M}_{N}(x)= \begin{cases}1 & \text { if } \min \left(\rho_{\mathrm{SE}}^{\varepsilon}(x), \rho_{\mathrm{SE}}^{\delta}(x)\right)>0 \\ 0 & \text { otherwise. }\end{cases}
$$

However, we note that other impulse detectors have been proposed in [19, 55, 44, 54] (see also the review [23]) and can be used for $\mathbf{M}_{N}$. Images contaminated with salt-and-pepper noise in range from $20 \%$ to $95 \%$, with increment steps of $5 \%$ are considered. In the case of colour images, a channel-independent salt-and-pepper noise was simulated according to the following rule [3]: the value of pixels was replaced by 0 with probability $\xi / 2$ and replaced by 1 with probability $\xi / 2$ with $\xi \in[0,1]$. Firstly, numerical experiments are carried out to demonstrate the performance of our proposed conditional toggle mapping for noise removal in standard test images (Lena, Goldhill, Cameraman, and Grey-Boat). Additionally, examples in colour images from the Kodak PhotoCD dataset are also included. Specifically, in applying our approach to colour images, we use a marginal approach where the conditional toggle mappings are applied to the different channels independently. The selection of an optimal colour space is an open problem but it is beyond the scope of this paper. Results of the proposed conditional toggle mapping by using the mask $\mathbf{M}_{N}$ are compared with three wellknown algorithms: Decision based Filtering [43], Progressive Switching Median Filter [51] and Classical Median Filter [12]. For quantitative comparison, we compute the peak signalto-noise ratio (PSNR). The PSNR in decibels is used to assess the strength of the filtered image defined as:

$$
P S N R=10 \log _{10} \frac{255^{2}}{1 / N^{2}|| \mathbf{I}_{\text {rest }}-\mathbf{I} \|^{2}}
$$

where $\mathbf{I}_{\text {rest }}$ is the restored image by a given operator and $N$ is the number of pixels in the image $\mathbf{I}$. This is used to measure the quality of an image.

The PSNR values resulting from various experiments are shown in Fig. 9. From these curves, it can be easily observed that the proposed conditional toggle mapping outperforms the other filtering schemes in noise level greater than $50 \%$. We remark that the results reported in Fig. 9 are the average of results of 25 repeated experiments. Furthermore, a subjective visual result of the noise reduction is presented in Figs. 10-11-12-13-14 for different noise densities.

We compared our approach with two state-of-the-art powerful denoising techniques on the Set-24 dataset [5]: Non-local means [4] and Kernel Regression [46]. The idea in Nonlocal means $[4,2]$, is to take advantage of self-similarities in images by comparing local 
neighbourhoods (patches) across the whole image. To deal with salt-and-pepper noise, the filtered pixel value is estimated as a weighted median of the pixels whose neighbourhoods are the most similar to the neighbourhood of the one to be denoised. Kernel Regression [46] is roughly based on the idea of a robust adaptive fitting of the image by using kernel functions. The kernel regression framework provides a rich mechanism for computing pointwise estimates of the regression function with minimal assumptions about global signal or noise models. The filtering result is a weighted mean from this local adaptive regression problem. Some popular existing methods as bilateral filter [47], are special cases of the kernel regression framework [6]. Table 1 summarises the results for the experiments. For the latter, we include the average PSNR over the set of images (as done also for the results reported in [28]). From the quantitative results show that for the heavily noisy data set (salt-and-pepper noise larger than 50\%) the proposed approach works better than non-local techniques [2][46]. However, these approaches work better when the noise level is less than $50 \%$. The authors suppose that this fact may due to two factors. On the one hand, the estimation step in non-local techniques is clearly affected by the lack of information (Noise level greater than 50\%). On the other hand, the proposed approach is "myope" in the sense that information considered in spatial neighbours is not greater than the 8-connectivity for a given pixel. However, that allows to deal with noise level higher that $90 \%$ as it is shown in Table 1.

Secondly, we compare our results to those of [5],[28]. The scheme of [28] was selected for comparison as it presents, to the best of knowledge, the best published results for saltand-pepper noise available in the literature. The performance of the proposed approach is significantly lower for the noisy level which have been considered in the published works, namely, iDUDE and regularisation based denoisers. However, our approach has as unique parameter the spatial connectivity (induced by the structuring element $\mathrm{SE}$ ) and it can be easily implemented using classical dilation operation. We remark that both [28] and [5] start with a traditional median filter, where the window size is related with noise level of the image. Our approach could be used instead of the traditional median filter to avoid that parameter in the algorithm. Once again, a remarkable point concerning the performance of the proposed approach is the fact that the difference in PSNR between the noise levels $50 \%$ and $95 \%$ is lower than 5 decibels, which shows the stability of the method with respect to the noise level.

\subsection{Application to hierarchical morphological segmentation}

The third application that we present is the improvement of quality of the hierarchical image segmentation by conditional toggle mapping in high resolution remote sensing. A complete description of this problem can be found in [41]. Hierarchical image segmentation is a very natural manner since the target has different meanings according to object size, i.e., from tile to building there are an entire range of scales. However, objects transitions depend on orien- 


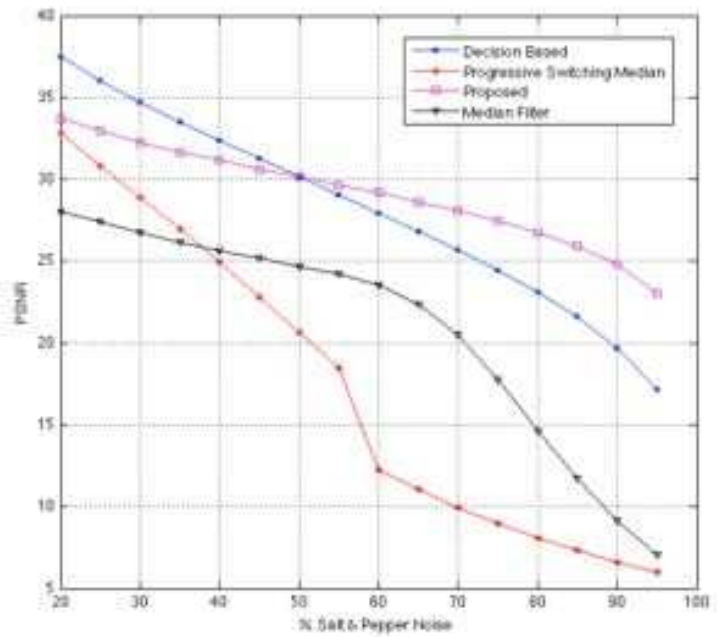

(a) Lena Grey

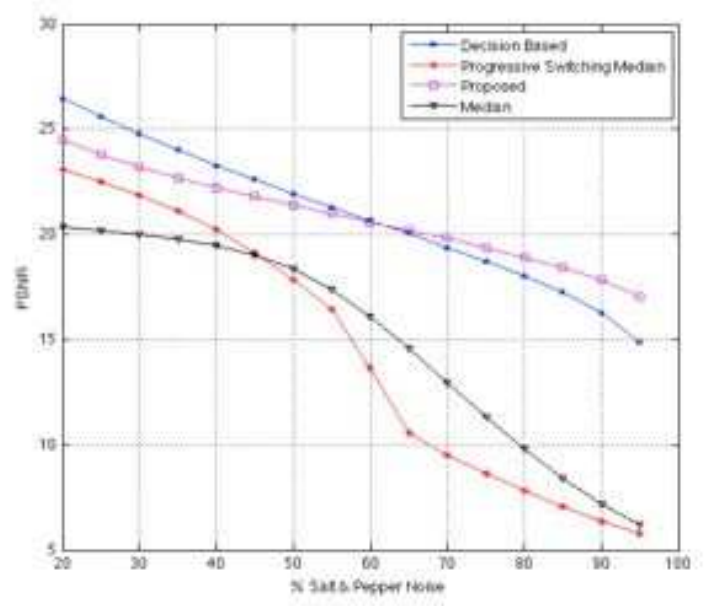

(c) Baboon Color

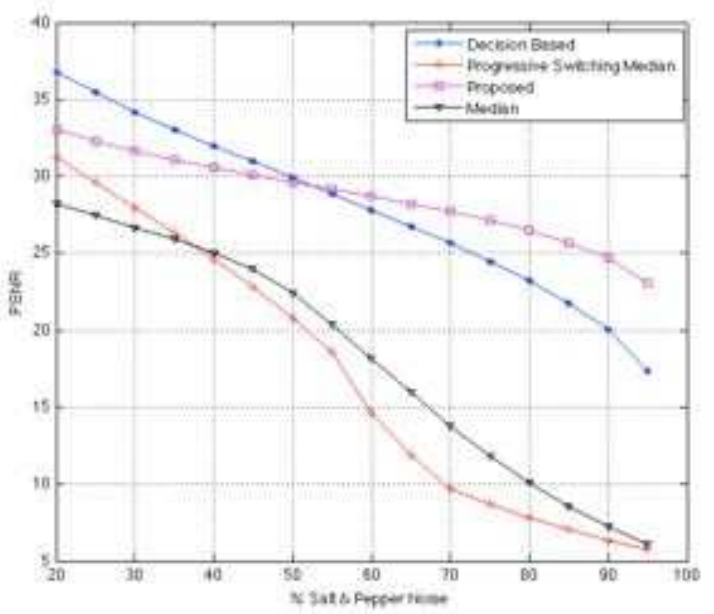

(b) Lena Color

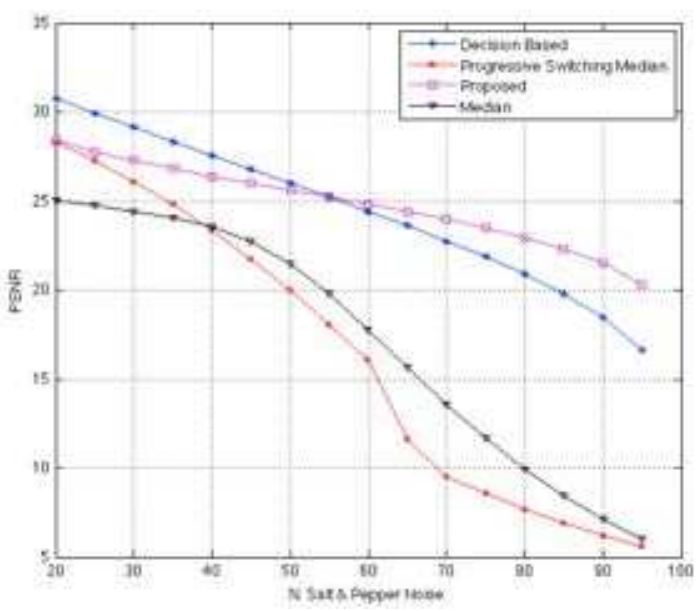

(d) Boat Color

Figure 9: Experimental results in PSNR for grey and colour Lena, Baboon and Boat at various noise levels for different approaches: Decision based [43], Progressive Switching Median Filter [51], and Classical Median Filter [12]. 


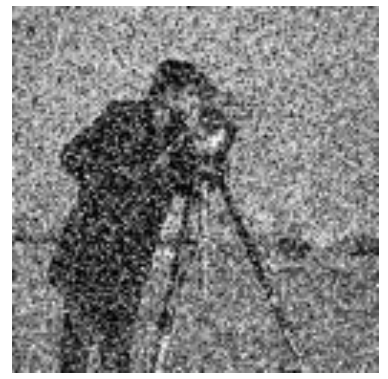

(a) $\mathrm{PSNR}=9.066$

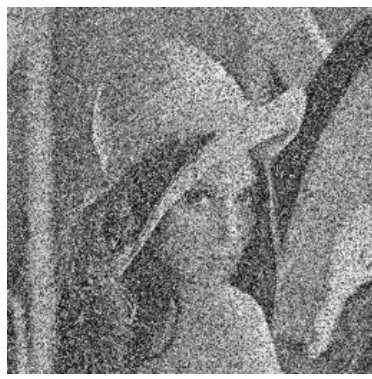

(b) $\mathrm{PSNR}=9.408$

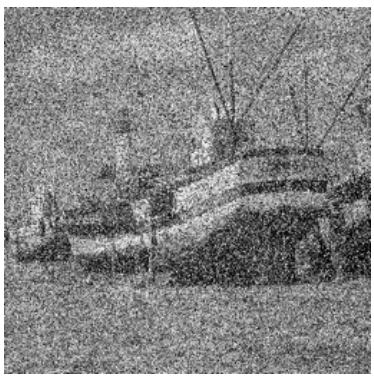

(c) $\mathrm{PSNR}=9.469$

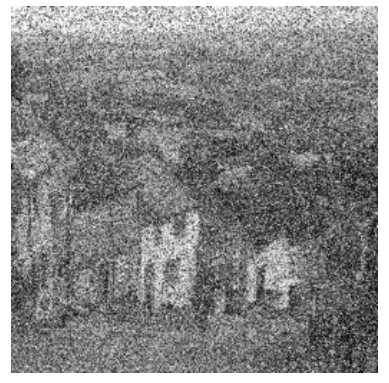

(d) $\mathrm{PSNR}=9.317$

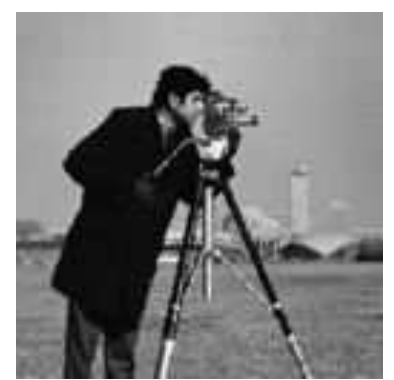

(e) $\mathrm{PSNR}=24.847$

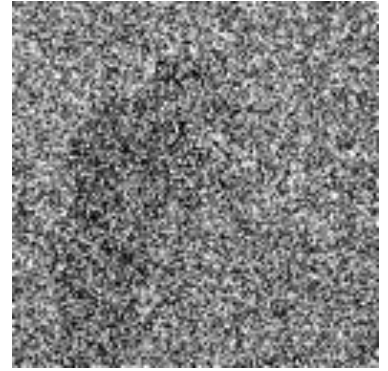

(i) $\mathrm{PSNR}=6.054$

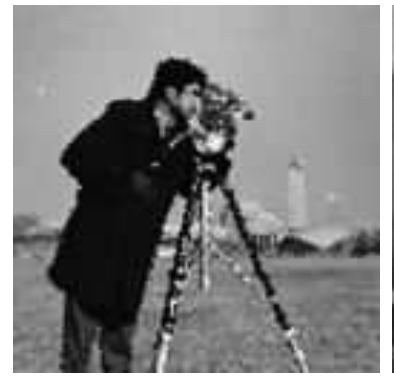

(m) $\mathrm{PSNR}=21.13$

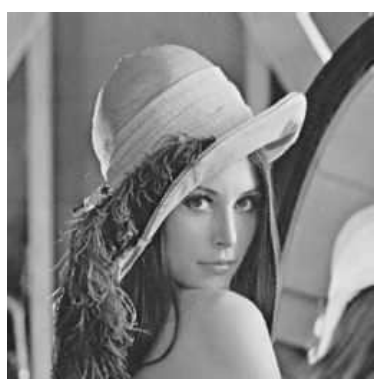

(f) $\mathrm{PSNR}=29.245$

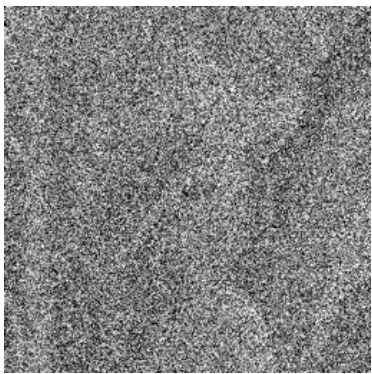

(j) $\mathrm{PSNR}=6.410$

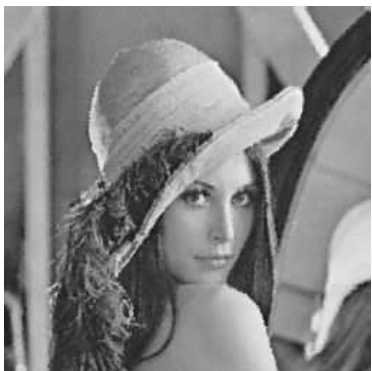

(n) $\mathrm{PSNR}=25.822$

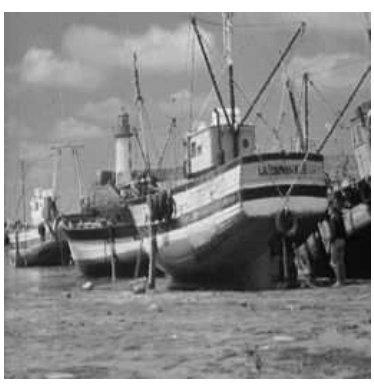

(g) $\mathrm{PSNR}=28.128$

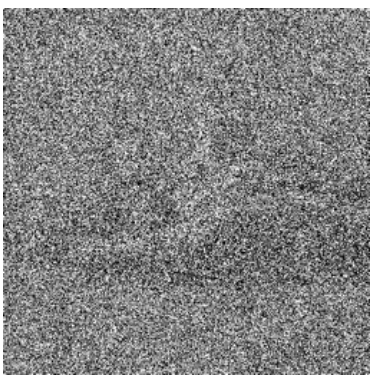

(k) $\mathrm{PSNR}=6.443$

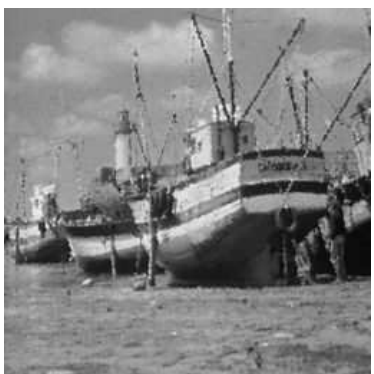

(o) $\mathrm{PSNR}=24.096$

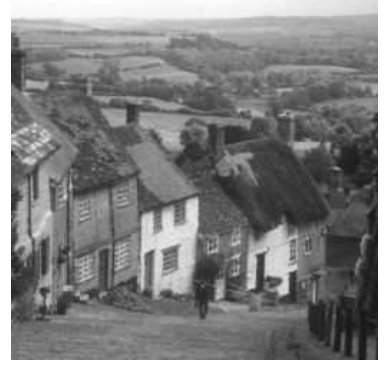

(h) $\mathrm{PSNR}=25.520$

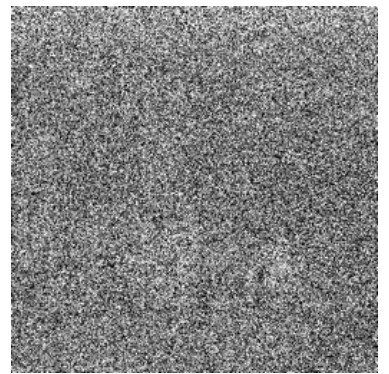

(l) $\mathrm{PSNR}=6.313$

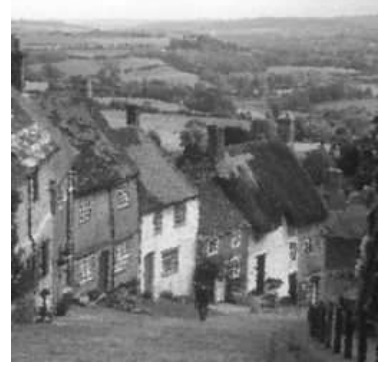

(p) $\mathrm{PSNR}=23.521$

Figure 10: Example of noise removal by proposed method. Images contaminated by salt-andpepper noise. (a)-(d) Noisy images (40\%.) (e)-(h) Results restored by using the conditional toggle mapping. (i)-(l) Noisy images (80\%.). (m)-(p) Image restored by the conditional toggle mapping. 


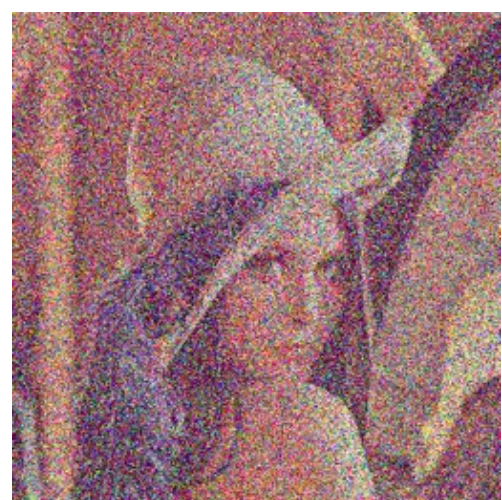

(a) $\mathrm{PSNR}=8.180$

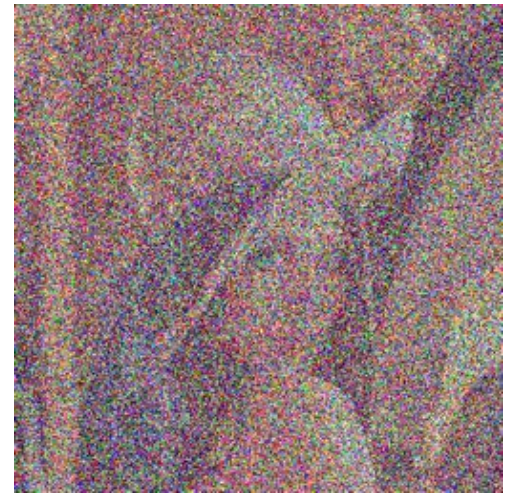

(d) $\mathrm{PSNR}=6.722$

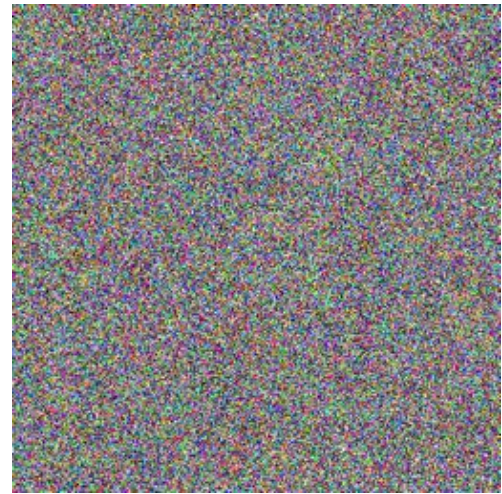

(g) $\mathrm{PSNR}=5.399$

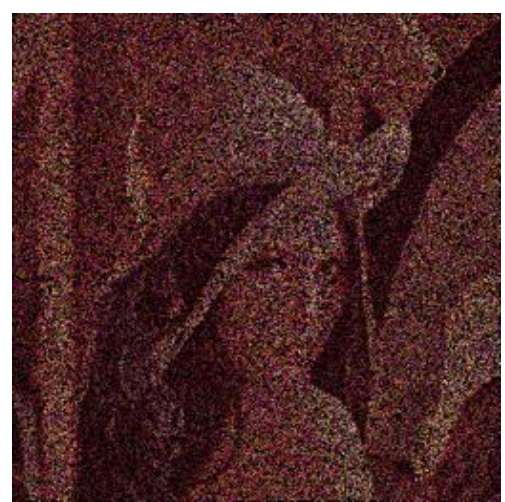

(b) $\mathrm{PSNR}=6.883$

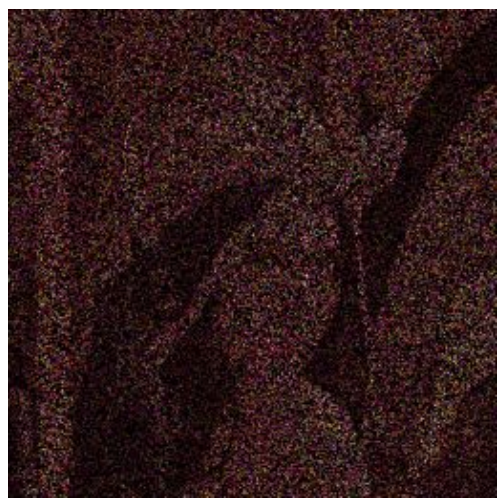

(e) $\mathrm{PSNR}=6.064$

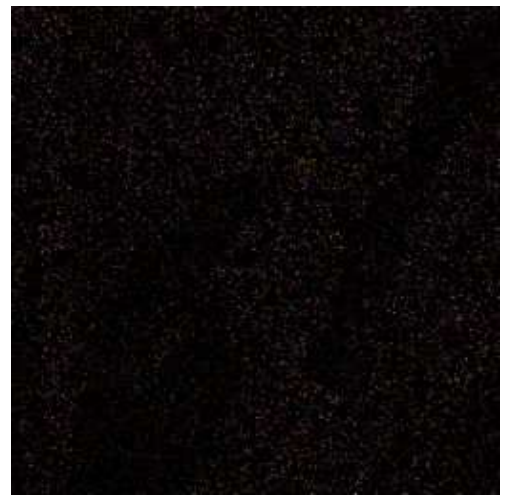

(h) $\mathrm{PSNR}=5.271$

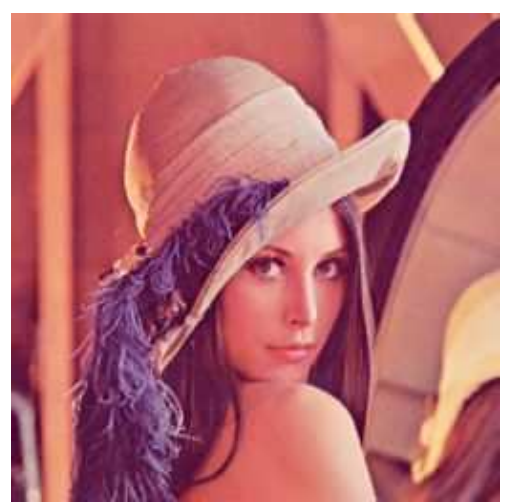

(c) $\mathrm{PSNR}=29.646$

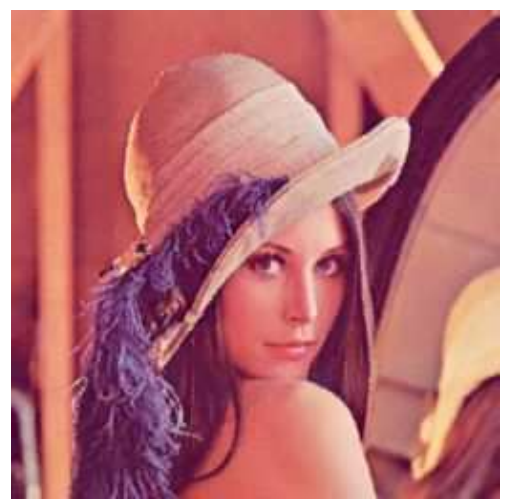

(f) $\mathrm{PSNR}=27.702$

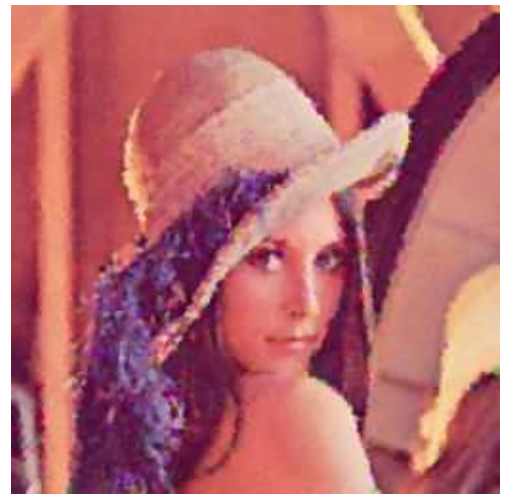

(i) $\mathrm{PSNR}=23.119$

Figure 11: Example of noise removal by proposed method. Images contaminated by marginal salt-and-pepper noise. (a)-(c) Experiment (50\%.) (d)-(f) Experiment (70\%). (g)-(i) Experiments (95\%.). 


\begin{tabular}{|c|c|c|c|c|c|}
\hline \% Noise & CHN05 [5] & iDUDE [28] & NL-Medians[4] & Kernel-Regression[45] & Conditional Morphology \\
\hline $30 \%$ & 34.5 & 35.1 & 27.93 & 28.94 & 26.81 \\
\hline $50 \%$ & 31.1 & 31.6 & 23.56 & 24.53 & 25.29 \\
\hline $70 \%$ & 28.1 & 28.6 & 22.28 & 21.29 & 24.28 \\
\hline $75 \%$ & 27.3 & N/A & 21.28 & 20.78 & 23.92 \\
\hline $80 \%$ & N/A & N/A & 19.90 & 18.65 & 23.47 \\
\hline $90 \%$ & N/A & N/A & 14.79 & 13.60 & 22.08 \\
\hline $95 \%$ & N/A & N/A & 10.09 & 9.93 & 20.69 \\
\hline \hline
\end{tabular}

Table 1: Comparison with state of the art in salt-and-pepper noise removal. CHN05 is median based approach with detail-preserving regularisation from [5], iDUDE is the application of optimal Bayes denoising rule on a context-conditioned sample probability distribution from [28]. Last column includes the results of the proposed conditional morphology. N/A is an abbreviation from not available.

tation, so it can alter segmentation results. Hierarchical segmentations are not subject to ties but, they are sensitive to the presence of intermediate value between object in the scene. This sensitivity is referred to as the chaining throughout transitions [41]. Basically, this negative effect can be perceived in different ways, for instance, when by small changes of the hierarchy parameter the segmentation becomes either big regions or a stream of small connected components located in transitions of the image. To make easier the result interpretation, we limited ourselves to the simplest case of connectivity, i.e., hierarchical segmentation based on $\alpha$-connectivity from [39].

\subsection{1 $\alpha$-connectivity}

$\alpha$-connectivity produces nested partitions with successive degree of coarseness by changing the connectivity threshold $[39,41]$. Given two pixels $x$ and $y \in \mathbb{E}$, they are $\alpha$-connected if there exist a path $\mathcal{P}$ going from $x$ to $y$ such that the dissimilarity $(d)$ between any two successive pixels of this path does not exceed the value $\alpha$, i.e.,

$$
\begin{aligned}
& \mathrm{CC}_{\alpha}(x)=\{x\} \cup\left\{y \mid \exists \mathcal{P}\left\langle x=p_{1}, p_{2}, \ldots, p_{m-1}, p_{m}=y\right\rangle, m>1,\right. \\
& \left.d\left(p_{i}, p_{j}\right) \leq \alpha \quad \forall i, j \quad 1 \leq i, j \leq m\right\}
\end{aligned}
$$

Basically, (16) is equivalent to the single linkage clustering method [13] considering finite similarities only among horizontal and vertical neighbours (4-neighbours or also known as direct-neighbour [12]). Additionally, (16) is equivalent to quasi-flat zones in the grey level case with dissimilarity set to absolute difference [26]. In the sequel, $\mathrm{CC}_{\alpha}(\mathbf{I})$ denotes the segmentation by (16) of the image I. Fig. 8 (b), (d),(f), and (h) show the flat zones (0connectivity), for the synthetic example presented in the preliminary subsection. Note that $\alpha$-connectivity (16) has the drawback that if two distinct image objects are separated by 
one or more transitions going in steps having dissimilarity value than or equal to $\alpha$, they appear within the same $\mathrm{CC}_{\alpha}$. That can be illustrated from Fig.8(c) or (e) where if the parameter $\alpha=0$ many one-pixel regions appear in the corner of the object and if $\alpha \geq 3$, we obtain only one region due to the presence of chaining throughout transitions in the image. We evaluate the performance of conditional toggle mapping as a pre-filtering to avoid these unpleasant transitions. A question of considerable interest in image processing, specially in digital remote sensing is: How well can image enhancement algorithms avoid this unpleasant effect in hierarchical image segmentation?

\subsubsection{Evaluation}

Firstly, we would like to remark that there is no standardised way to measure the quality of the algorithms to confront this problem. So, we start with a qualitative evaluation through the visual appearance of the segmentations before/after the application of (11) by using the mask in (13). We show the results of classical $\tau_{\mathrm{SE}}^{\infty}$ in (Fig. 15(b)) and proposed $\tilde{\tau}_{\mathrm{SE}}^{\infty}$ in Fig. 15(c) for the high resolution image in Fig. 15(a). We also include two examples in hierarchical segmentation based on $\mathrm{CC}_{\alpha}$. For $\mathrm{CC}_{\alpha=4}$ in Fig. 16 and $\mathrm{CC}_{\alpha=8}$ in Fig. 17. Four zoomed boxes are included to make easier the interpretation. Coloured frames overlaid on the large image show what portions are magnifying. Note that the segmentations from the original image Fig. 16(a) and 17(b) have a huge main connected component caused by chaining throughout transitions effect on the original image. Both classical and conditional toggle mapping manages to avoid this unpleasant effect. Additionally, the number of connected components are reported per segmentation. We can observe how main structures on the image are preserved even if the number of connected component have been drastically reduced, i.e., from 130, 077 to 22, 461 in Fig. 16 and 53, 506 to 8, 196 in Fig. 17. A complete comparison for values of $\alpha$ in $[0, \ldots, 30]$ have been included in Fig. 18(a). Secondly, we give a quantitative comparison of segmentations. By using the mean of $\mathbf{I}(\mathbf{x})$ each $\mathrm{CC}_{\alpha}$ we recover an image and the PSNR (15) is calculated for values of $\alpha=[0,4, \ldots, 30]$ with respect to the original image. Fig. 18(b) shows that segmentation in classical and conditional toggle mapping provides better PSNR in comparison with segmentation with equivalent number of connected components on the original image. The performances of conditional and classical toggle mapping are comparable but the first one can be computed at least seven times faster than classical one.

\section{Conclusions and Perspectives}

In this paper, we have provided three main contributions:

- Mask-based morphology: We have introduced a mask based mathematical morphology operators by revisiting shock filters based on erosions and dilations and extending their 
definition to take into account the prior definition of a mask of pixels that should not be altered.

- Conditional operators as morphological filters: We proof the mildly counterintuitive results that conditional dilation and erosion are morphological filters [35], thanks to the fact that conditional operators defined in this paper, can be considered in the framework of adaptive morphology [32].

- Flexible formulation: Proposed approach is based on a binary mask $\mathbf{M}$ which makes it possible to address different problems in image processing.

In our experimental section, examples of application in edge enhancement, salt-and-pepper denoising and prefiltering in hierarchical segmentation have been analysed in detail. In the first case, a mask based shock filter by generalising the original Kramer formulation with constrains on the set on pixels marked by the binary image $\mathbf{M}$ had been formulated. It is robust to halo problems, with convergence in a few number of iterations. Recommendations for future work includes the incorporation of adaptive dilation on the operator $\tilde{\tau}_{\mathrm{SE}}$.

In the second case, the proposed denoising filter also shows consistent and stable performance across a wide range of noise densities from $10 \%$ to $95 \%$. In fact, the difference in PSNR after reconstruction between the noise levels $50 \%$ and $95 \%$ is lower than 5 decibels.

In the third case, we have illustrated how our approach can help to improve the quality of morphological segmentation as the classical toggle mapping, but it can be computed in a small number of iterations allowing a good speed-up in practical applications. We are currently studying the properties of approaches where the mask image serves as a series of seeds in the framework of seeded region growing (see example in [40]). Additionally, interesting future work includes:

1. Optimal selection of the shape/size for the SE.

2. Compression based on conditional morphology.

3. Extension to high dimensional images by using vector morphology [48, 49] instead of the marginal ordering scheme adopted in this paper.

\section{References}

[1] Alvarez, L., Mazorra, L.: Signal and image restoration using shock filters and anisotropic diffusion. SIAM Journal of Numerical Analysis 31, 590-605 (1994)

[2] Baudes, A., Coll, B., Morel, J.: A review of image denoising algorithms, with a new one. Multiscale Modeling and Simulation 4(2), 490-530 (2005)

[3] Boncelet, C.: Image noise models. In: A.C. Bovik (ed.) Handbook of image and video processing, pp. 325-335. New York: Academic (2000) 
[4] Buades, A., Coll, B., Morel, J.M.: A review of image denoising algorithms, with a new one. Multiscale Modeling Simulation 4, 490-530 (2005)

[5] Chan, R., Chung-Wa, H., Nikolova, M.: Salt-and-pepper noise removal by mediantype noise detectors and detail-preserving regularization. IEEE Transactions on Image Processing 14(10), 1479-1485 (2005)

[6] Chatterjee, P., Milanfar, P.: Is denoising dead? IEEE Transactions on Image Processing 19(4), $895-911(2010)$

[7] Diop, E., Angulo, J.: Spatially adaptive pdes for robust image sharpening. In: Proc. Int. Conf. Image Processing (ICIP), pp. 949-952 (2012)

[8] Elder, J., Zucker, S.: Local scale control for edge detection and blur estimation. IEEE Transactions on Pattern Analysis and Machine Intelligence 20(7), 699-716 (1998)

[9] Gierz, G., Hofmann, K.H., Keimel, K., Lawson, J.D., Mislove, M., Scott, D.S.: A Compendium of Continuous Lattices. Springer-Verlag (1980)

[10] Gilboa, G., Sochen, N., Zeevi, Y.: Image enhancement and denoising by complex diffusion processes. IEEE Transactions on Pattern Analysis and Machine Intelligence 25(8), 1020-1034 (2004)

[11] Gilboa, G., Sochen, N.A., Zeevi, Y.Y.: Regularized shock filters and complex diffusion. In: Proceeding of the 7th European Conference on Computer Vision-Part I, ECCV '02, pp. 399-413. Springer-Verlag, London, UK (2002)

[12] Gonzalez, R., Woods, R.: Digital image processing. Prentice Hall (2008)

[13] Gower, J., Ross, G.: Minimum spanning trees and single linkage cluster analysis. Applied Statistics 18(1), 54-64 (1969)

[14] Grazzini, J., Soille, P.: Edge-preserving smoothing using a similarity measure in adaptive geodesic neighborhoods. Pattern Recognition 42, 2306-2316 (2009)

[15] Heijmans, H.J.A.M.: Morphological Image Operators, vol. 10. Academic Press (1994)

[16] Heijmans, H.J.A.M.: Composing morphological filters. IEEE Transactions on Image Processing 6(5), 713-724 (1997). DOI 10.1109/83.568928

[17] Heijmans, H.J.A.M., Nacken, P., Toet, A., Vincent, L.: Graph morphology. Journal of Visual Communication and Image Representation 3, 24-38 (1992)

[18] Heijmans, H.J.A.M., Ronse, C.: The algebraic basis of mathematical morphology. I. dilations and erosions. Comput. Vision Graph. Image Process. 50, 245-295 (1990) 
[19] Hwang, H., Haddad, R.A.: Adaptive median filters: new algorithms and results. IEEE Transactions on Image Processing 4, 499-502 (1995)

[20] Jochems, T.: Morphologie mathématique appliquée au contrôle industriel de pièces coulées. Ph.D. thesis, Ecole National Supérieure des Mines de Paris (1997)

[21] Keshet, R., Heijmans, H.J.A.M.: Adjunctions in pyramids, curve evolution and scalespaces. International Journal of Computer Vision 52, 139-151 (2003)

[22] Kramer, H.P., Bruckner, J.B.: Iterations of a non-linear transformation for enhancement of digital images. Pattern Recognition 7(1-2), 53-58 (1975)

[23] Lukac, R., Smolka, B., Martin, K., Plataniotis, K., Venetsanopoulos, A.: Vector filtering for color imaging. Signal Processing Magazine, IEEE 22(1), 74-86 (2005)

[24] Mallat, S.: A wavelet tour of signal processing, third edition: The sparse way, 3rd edn. Academic Press (2008)

[25] Maragos, P.: Lattice image processing: A unification of morphological and fuzzy algebraic systems. Journal of Mathematical Imaging and Vision 22, 333-353 (2005)

[26] Meyer, F.: The levelings. In: Proc. of the ISMM'98, pp. 199-206. Kluwer Academic Publishers, Norwell, MA, USA (1998)

[27] Meyer, F., Serra, J.: Contrasts and activity lattice. Signal Processing 16(4), 303-317 (1989). DOI 10.1016/0165-1684(89)90028-5

[28] Motta, G., Ordentlich, E., Ramírez, I., Seroussi, G., Weinberger, M.J.: The idude framework for grayscale image denoising. IEEE Transactions on Image Processing 20(1), 1-21 (2011)

[29] Osher, S., Rudin, L.: Feature-oriented image enhancement using shock filters. SIAM Journal on Numerical Analysis 27, 919-940 (1990)

[30] Osher, S., Rudin, L.: Shocks and other nonlinear filtering applied to image processing. In: Society of Photo-Optical Instrumentation Engineers (SPIE) Conference Series, vol. 1567, pp. 414-431 (1991)

[31] Rivest, J.F., Soille, P., Beucher, S.: Morphological gradients. Journal of Electronic Imaging 2(4), 326-336 (1993). DOI 10.1117/12.159642

[32] Roerdink, J.B.T.M.: Adaptivity and group invariance in mathematical morphology. In: International Conference of Image Processing, pp. 2253-2256. IEEE (2009). DOI 10.1109/ICIP.2009.5413983

[33] Roerdink, J.B.T.M.: personal communication (2012) 
[34] Schavemaker, J., Reinders, M.J.T., Gerbrands, J.J., Backer, E.: Image sharpening by morphological filtering. Pattern Recognition 33(6), 997-1012 (2000)

[35] Serra, J.: Image Analysis and Mathematical Morphology Volume 2: Theoretical Advances. Academic Press (1988)

[36] Serra, J.: Toggle mappings. In: From Pixels to Features, pp. 61-72. Simon, Ed., North Holland, Amsterdam (1989)

[37] Serra, J., Vincent, L.: An overview of morphological filtering. Circuits, Systems and Signal Processing 11(1), 47-108 (1992)

[38] Soille, P.: Beyond self-duality in morphological image analysis. Image Vision and Computing 23(2), 249-257 (2005)

[39] Soille, P.: Constrained connectivity for hierarchical image partitioning and simplification. IEEE Transactions on Pattern Analysis and Machine Intelligence 30(7), 1132-1145 (2008)

[40] Soille, P.: Constrained connectivity for the processing of very-high-resolution satellite images. Int. J. Remote Sens. 31(22), 5879-5893 (2010)

[41] Soille, P.: Preventing chaining through transitions while favouring it within homogeneous regions. In: Mathematical Morphology and Its Applications to Image and Signal Processing, Lecture Notes in Computer Science, vol. 6671, pp. 96-107. Springer Berlin / Heidelberg (2011)

[42] Soille, P., Grazzini, J.: Constrained connectivity and transition regions. In: Proc. of the ISMM'09, pp. 59-69. Springer-Verlag, Berlin, Heidelberg (2009)

[43] Srinivasan, K., Ebenezer, D.: A new fast and efficient decision-based algorithm for removal of high density impulse noises. IEEE Signal Processing Letters 14(3), 189-192 (2007)

[44] Taguchi, A., Matsumoto, T.: Removal of impulse noise from highly corrupted images by using noise position information and directional information of image. In: Proc. SPIE 4304, Nonlinear Image Processing and Pattern Analysis XII, pp. 188-196 (2001)

[45] Takeda, H., Farsiu, S., Milanfar, P.: Robust kernel regression for restoration and reconstruction of images from sparse, noisy data. In: Proc. Int. Conf. Image Processing (ICIP, pp. 1257-1260 (2006)

[46] Takeda, H., Farsiu, S., Milanfar, P.: Kernel regression for image processing and reconstruction. IEEE Transactions on Image Processing 16(2), 349-366 (2007) 
[47] Tomasi, C., Manduchi, R.: Bilateral filtering for gray and color images. In: Computer Vision, 1998. Sixth International Conference on, pp. 839 -846 (1998)

[48] Velasco-Forero, S., Angulo, J.: Supervised ordering in $\mathbb{R}^{p}$ : Application to morphological processing of hyperspectral images. IEEE Transactions on Image Processing 20(11), 3301-3308 (2011)

[49] Velasco-Forero, S., Angulo, J.: Random projection depth for multivariate mathematical morphology. J. Sel. Topics Signal Processing 6(7), 753-763 (2012)

[50] van Vliet, L., Young, I., Beckers, G.: A nonlinear Laplace operator as edge detector in noisy images. Computer Vision, Graphics, and Image Processing 45(2), 167-195 (1989). DOI 10.1016/0734-189X(89)90131-X

[51] Wang, Z., Zhang, D.: Progressive switching median filter for the removal of impulse noise from highly corrupted images. IEEE Trans. Circuits and Systems II: Analog and Digital Signal Processing 46(1), 78-80 (1999)

[52] Weickert, J.: Coherence-enhancing shock filters. In: Lecture Notes in Computer Science, pp. 1-8. Springer-Verlag (2003)

[53] Welk, M., Weickert, J., Galć, I.: Theoretical foundations for spatially discrete 1-d shock filtering. Image and Vision Computing 25, 455-463 (2007)

[54] Xu, H., Zhu, G., Peng, H., Wang, D.: Adaptive fuzzy switching filter for images corrupted by impulse noise. Pattern Recogn. Lett. 25(15), 1657-1663 (2004)

[55] Zhang, S., Karim, M.: A new impulse detector for switching median filters. IEEE Signal Processing Letters 9(11), 360-363 (2002) 


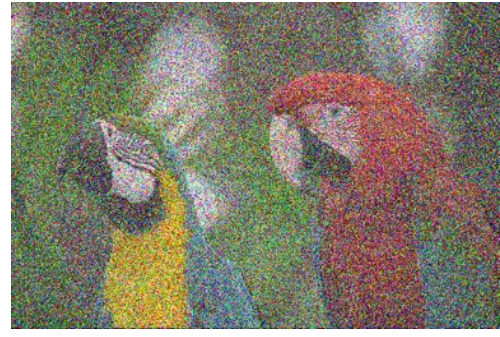

(a) $\mathrm{PSNR}=8.098$

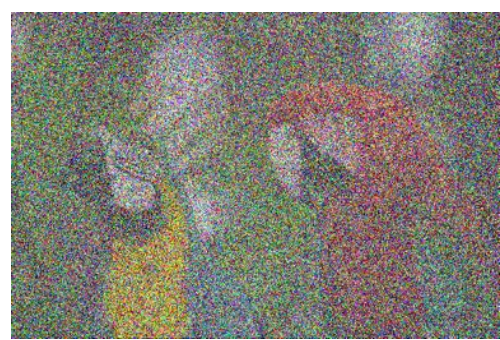

(d) $\mathrm{PSNR}=6.630$

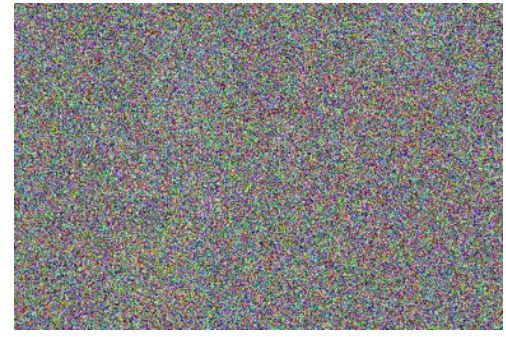

(g) $\mathrm{PSNR}=5.301$

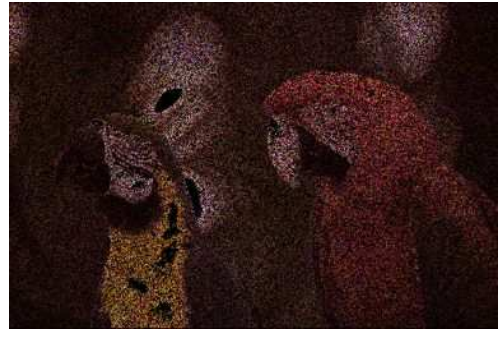

(b) $\mathrm{PSNR}=8.194$

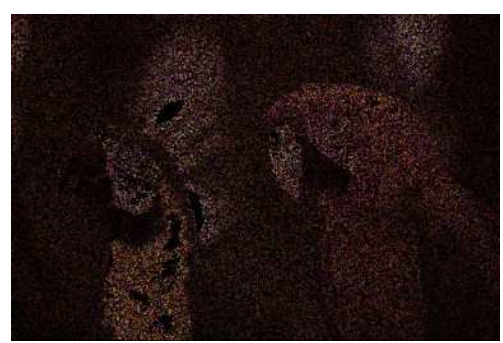

(e) $\mathrm{PSNR}=7.527$

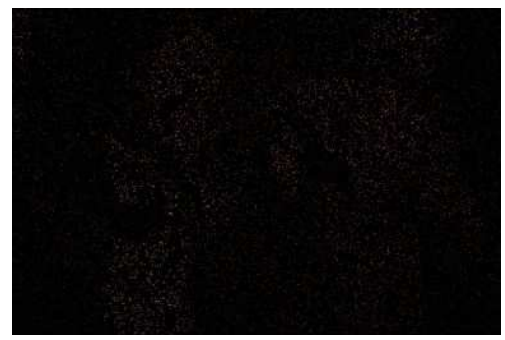

(h) $\mathrm{PSNR}=6.855$

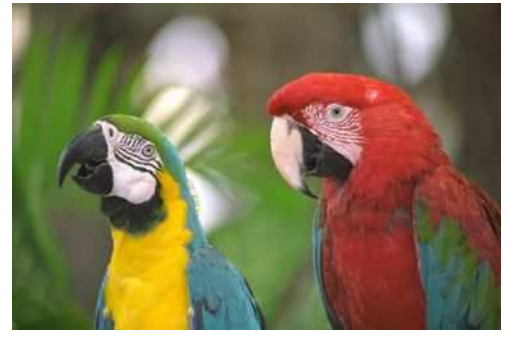

(c) $\mathrm{PSNR}=29.494$

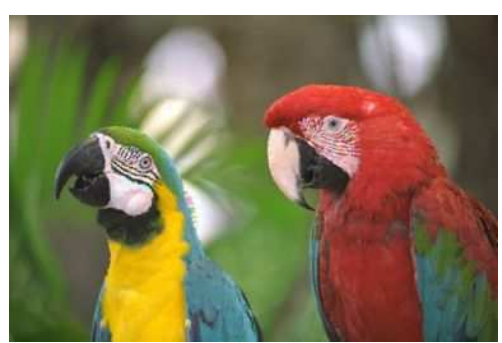

(f) $\mathrm{PSNR}=27.940$

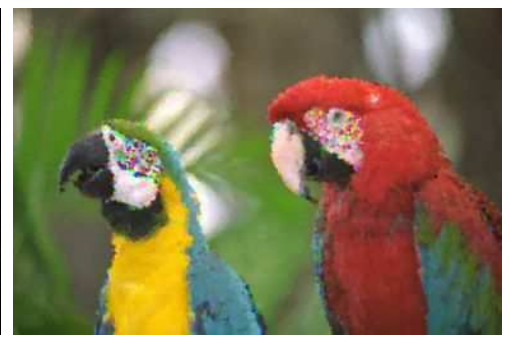

(i) $\mathrm{PSNR}=23.958$

Figure 12: Example of noise removal by proposed method.(a)-(c) Experiment (50\%). (d)-(f) Experiment (70\%). (g)-(i) Experiments (95\%.). First column shows images contaminated by marginal salt-and-pepper noise, second column includes mask image by using Eq. (14) and third column displays the result of the conditional toggle mapping. 


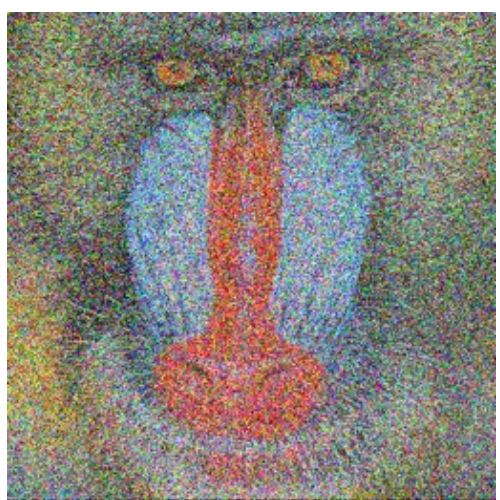

(a) $\mathrm{PSNR}=8.264$

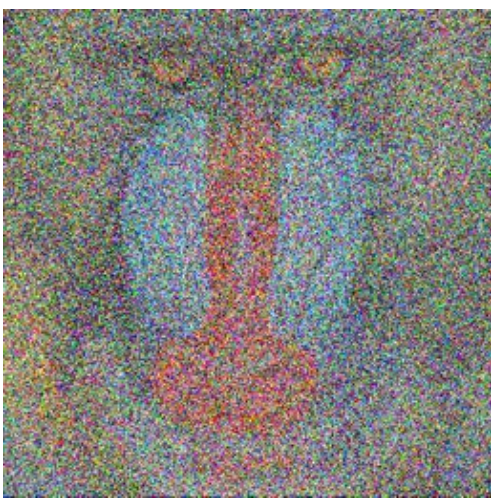

(d) $\mathrm{PSNR}=6.798$

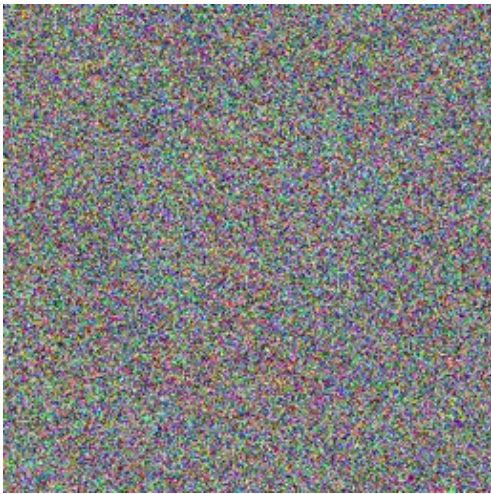

(g) $\mathrm{PSNR}=5.473$

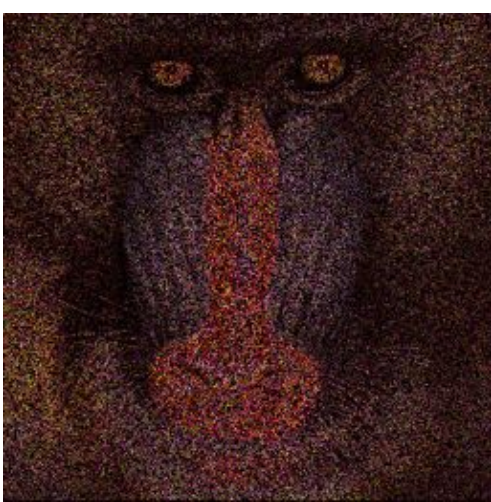

(b) $\mathrm{PSNR}=6.882$

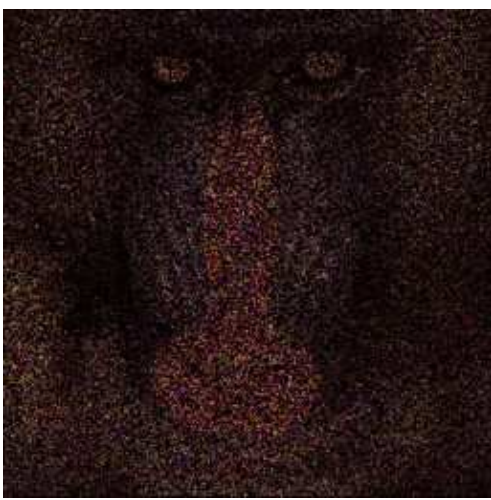

(e) $\mathrm{PSNR}=6.146$

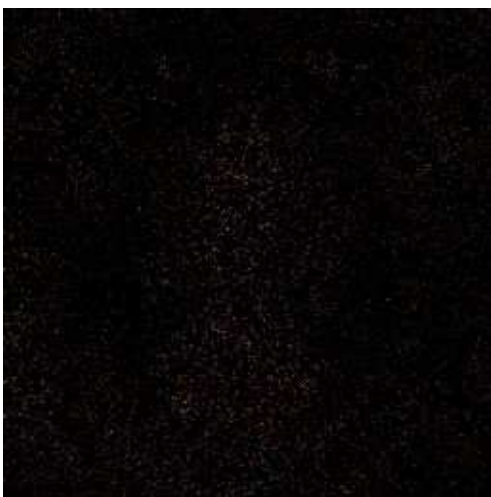

(h) $\mathrm{PSNR}=5.429$

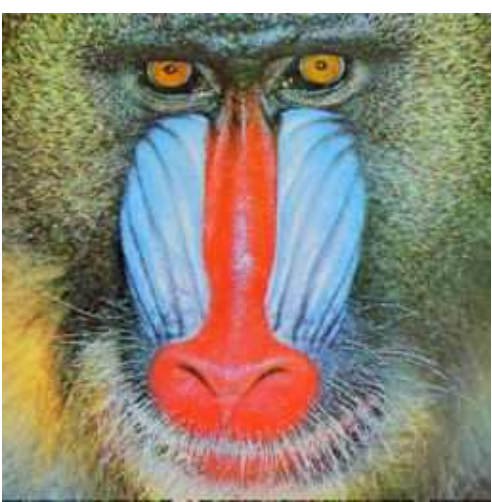

(c) $\mathrm{PSNR}=21.395$

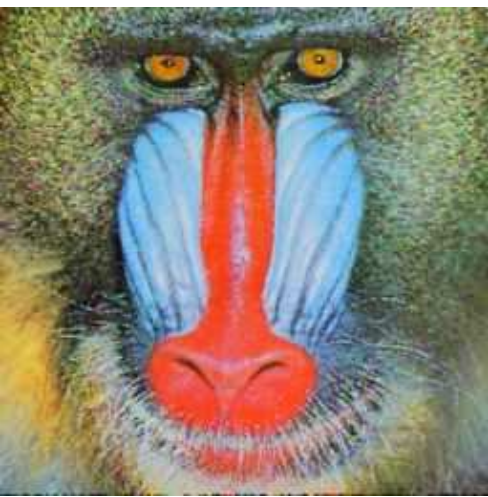

(f) $\mathrm{PSNR}=19.817$

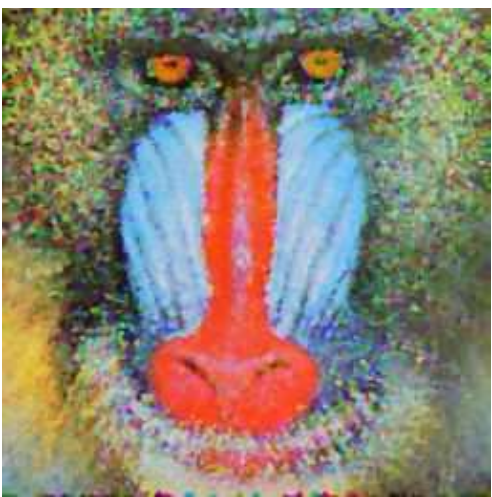

(i) $\mathrm{PSNR}=17.024$

Figure 13: Example of noise removal by proposed method. Images contaminated by marginal salt-and-pepper noise. (a)-(c) Experiment (50\%.) (d)-(f) Experiment (70\%). (g)-(i) Experiments (95\%.). 


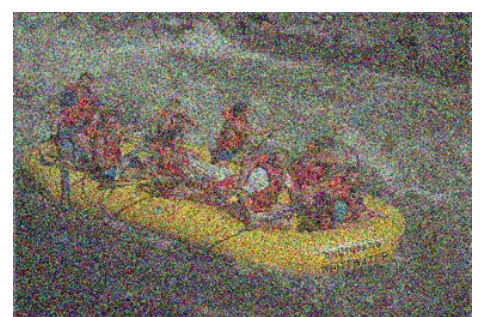

(a) $\mathrm{PSNR}=8.069$

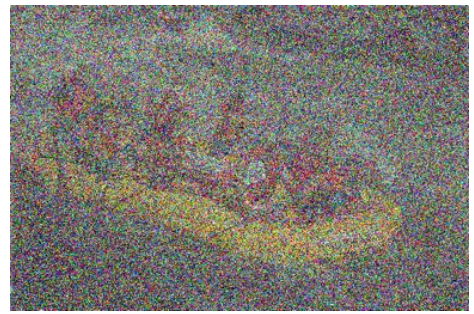

(d) PSNR $=6.598$

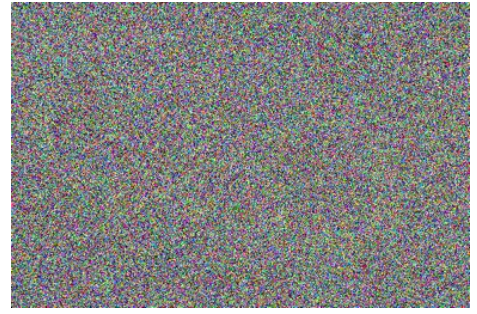

(g) $\mathrm{PSNR}=5.264$

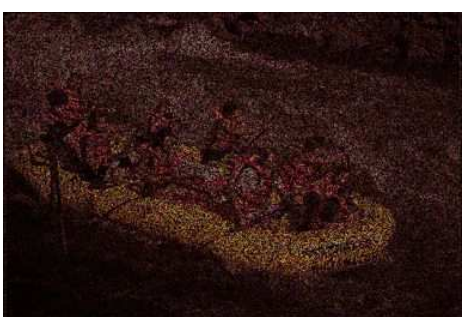

(b) $\mathrm{PSNR}=9.487$

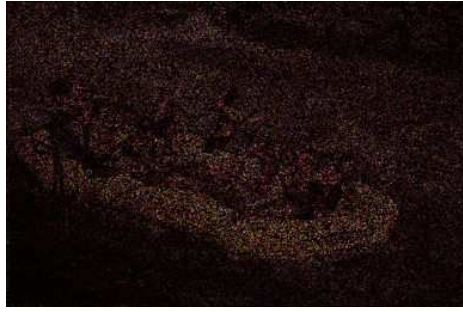

(e) $\mathrm{PSNR}=8.717$

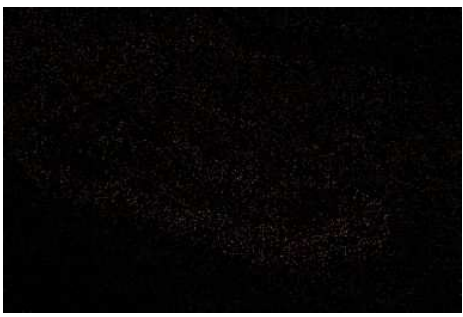

(h) $\mathrm{PSNR}=7.973$

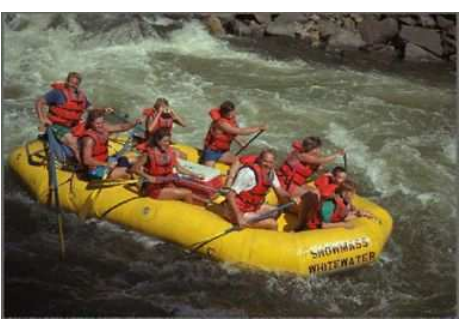

(c) $\mathrm{PSNR}=25.576$

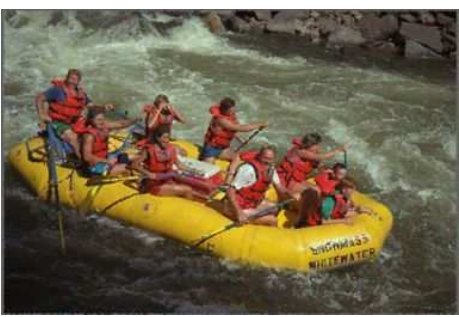

(f) $\mathrm{PSNR}=23.939$

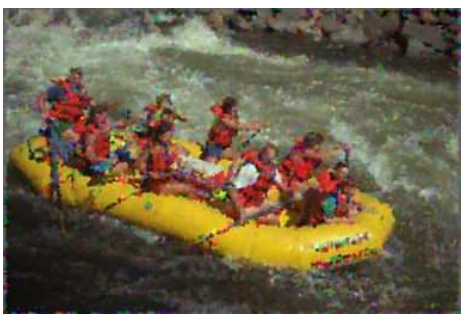

(i) $\mathrm{PSNR}=20.276$

Figure 14: Example of noise removal by proposed method. Images contaminated by marginal salt-and-pepper noise. (a)-(c) Experiment (50\%.) (d)-(f) Experiment (70\%). (g)-(i) Experiments (95\%.). 

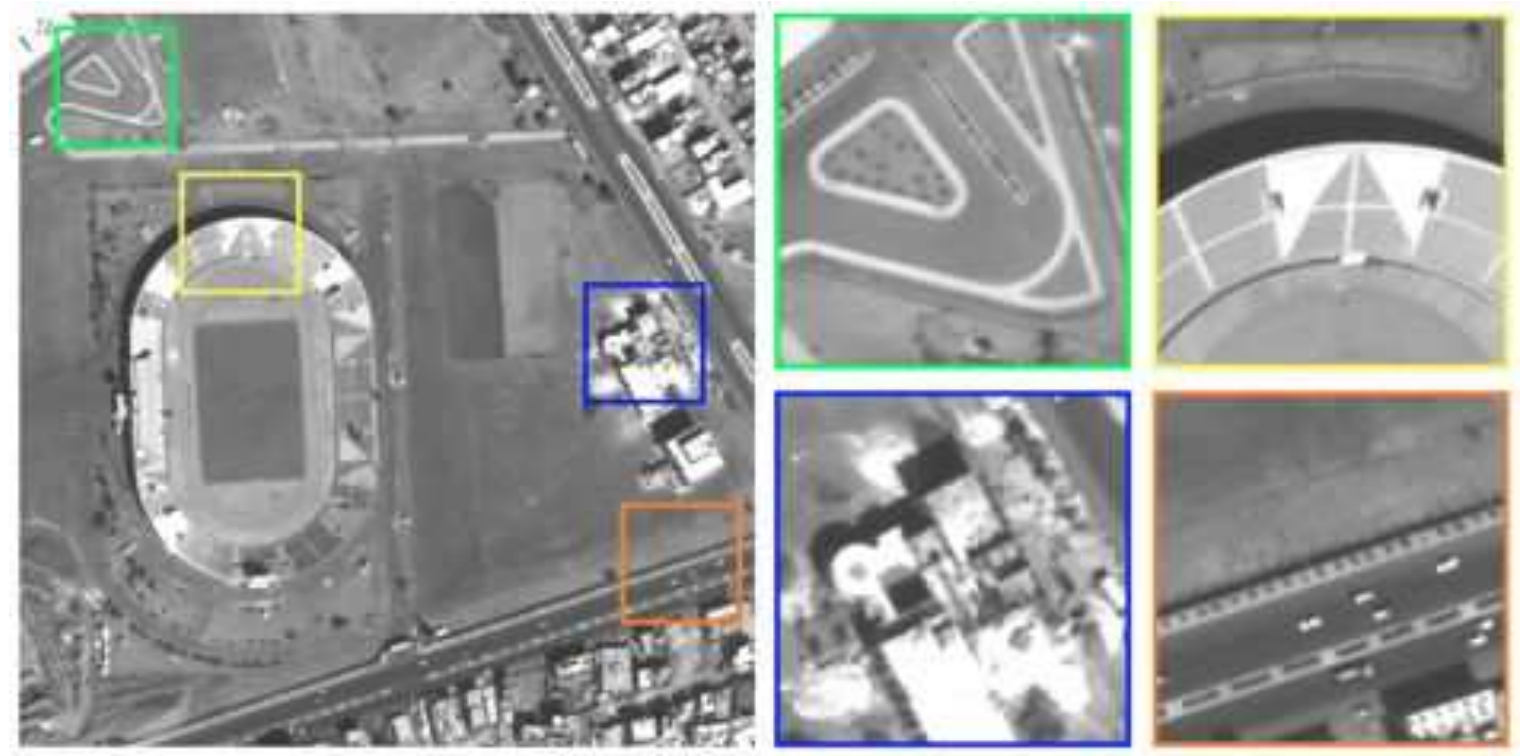

(a) Original image $\mathbf{I}$ is $1000 \times 1000$ pixels. It was extracted from a WorldView-2 satellite image (European Space Imaging) with spatial resolution of $50 \mathrm{~cm}$.
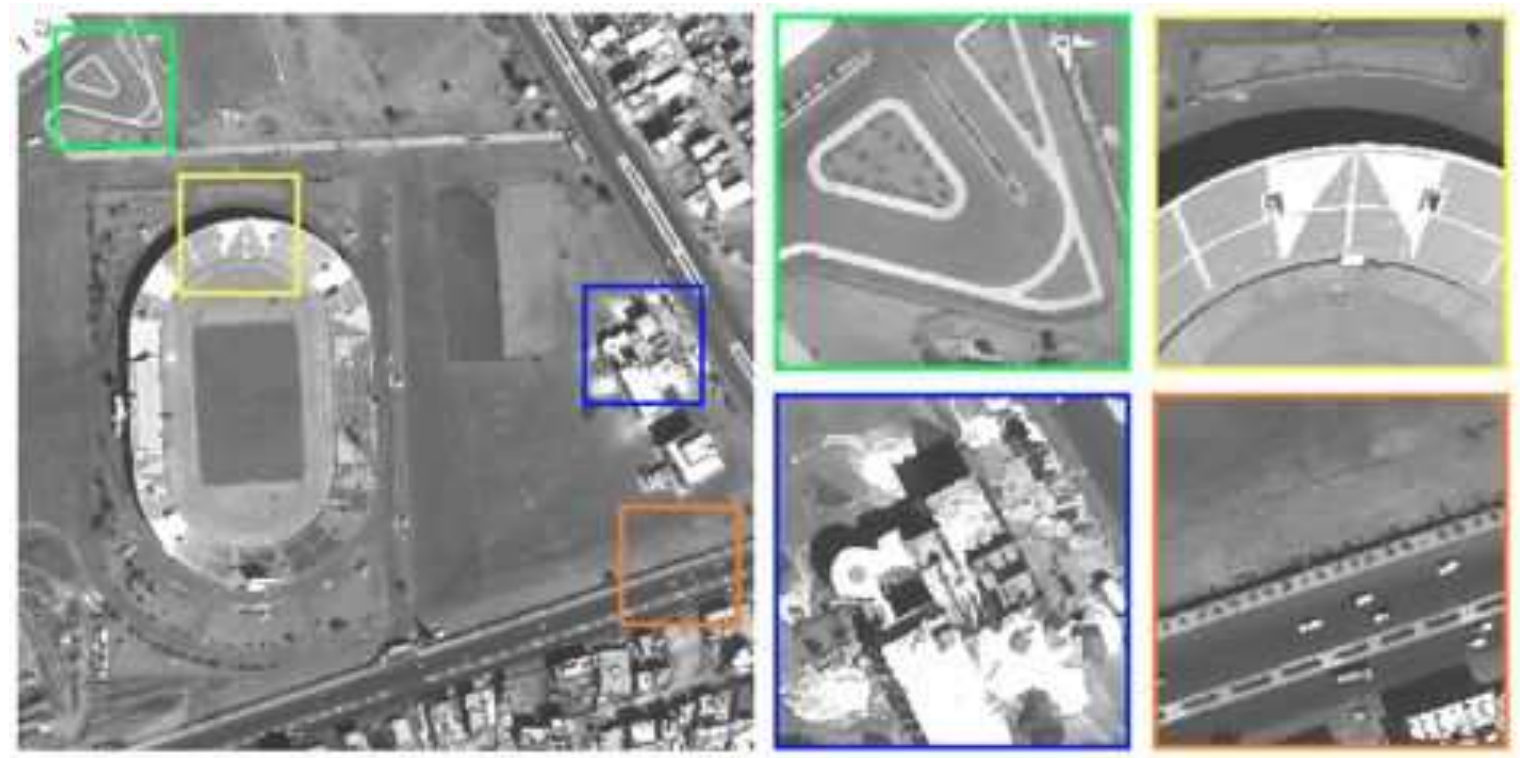

(b) Toggle Mapping $\left(\tau_{\mathrm{SE}}^{\infty}(\mathbf{I})\right)$ of (a). Computational time is $9.95 \mathrm{sec}$.
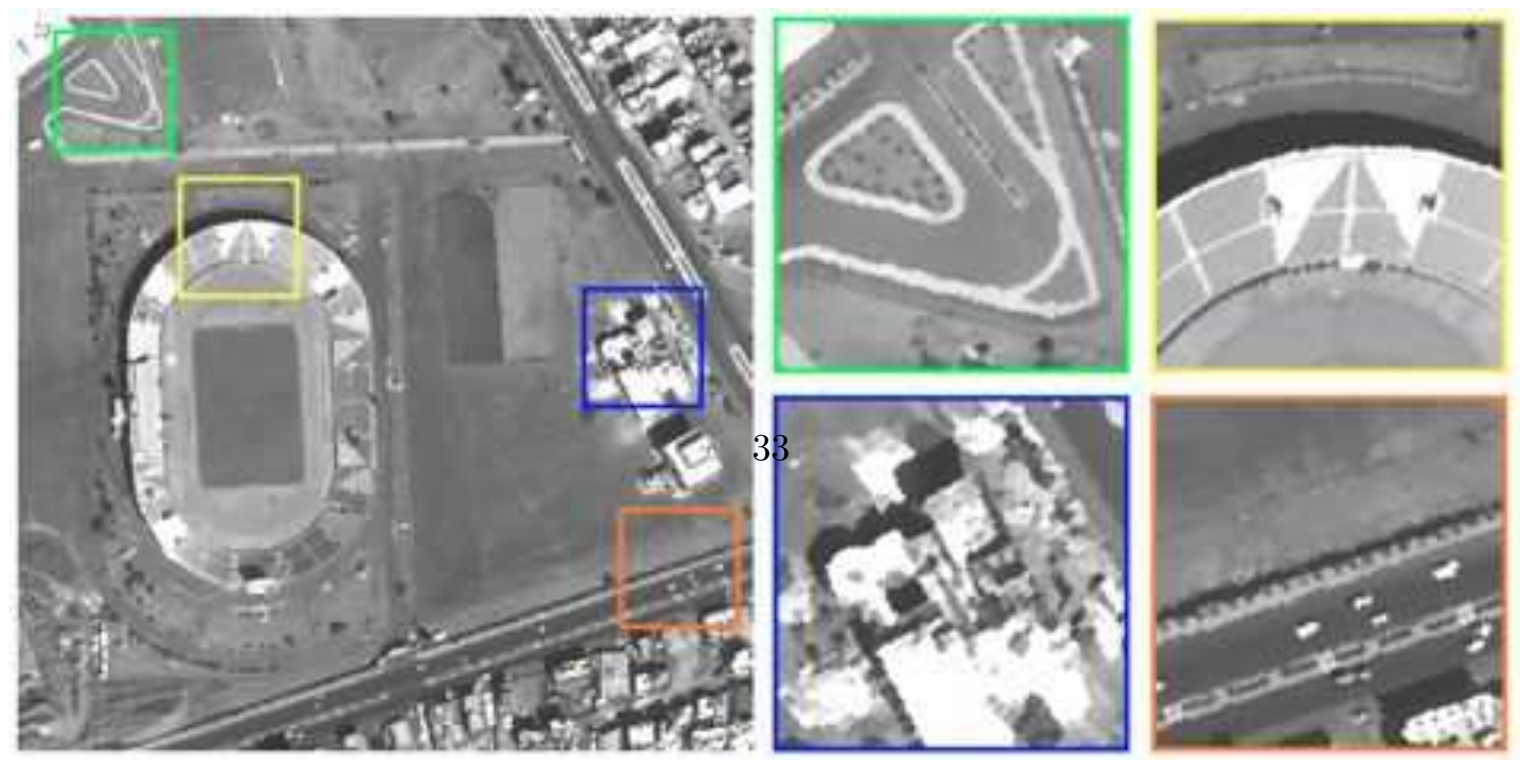

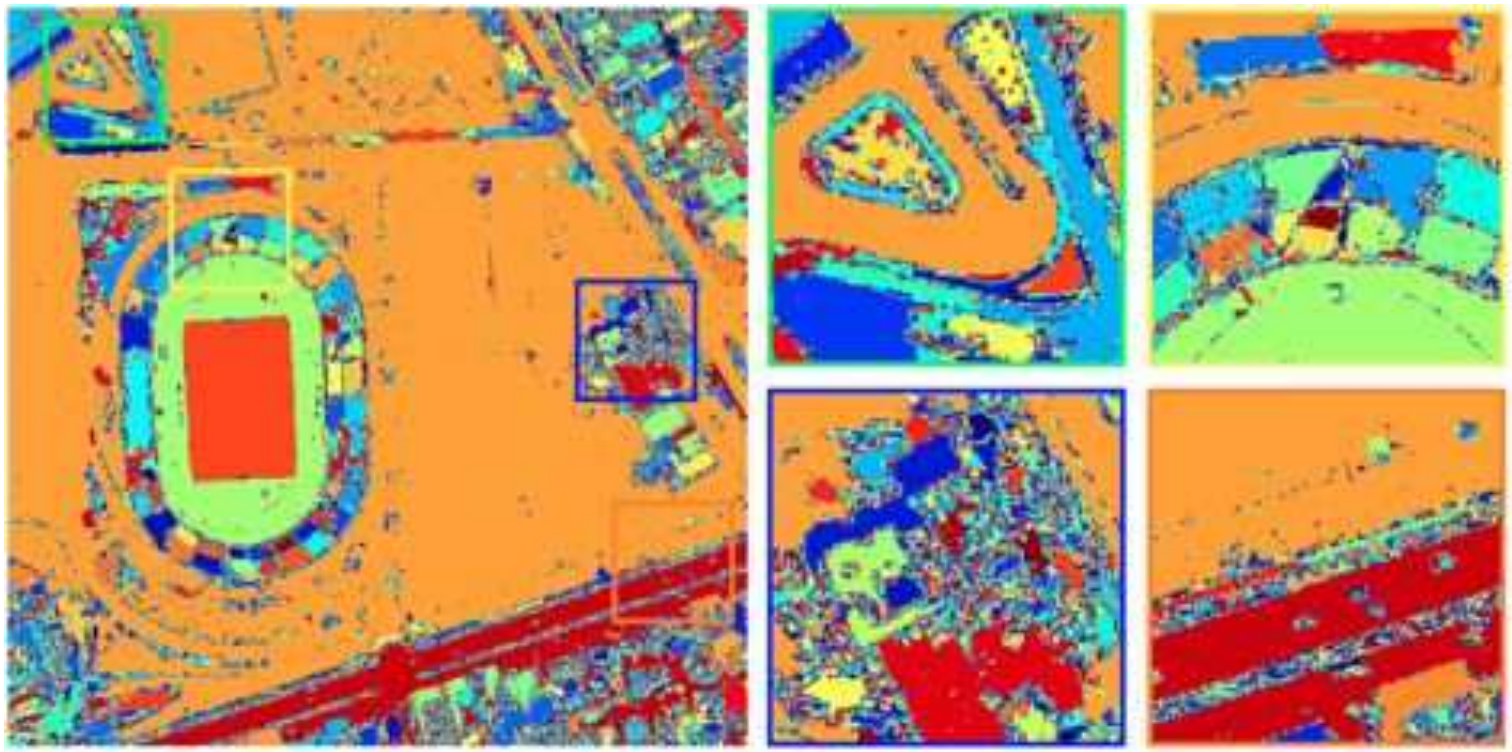

(a) $\mathrm{CC}_{\alpha}$ (I) from 15(a). \# CC $=130,077$
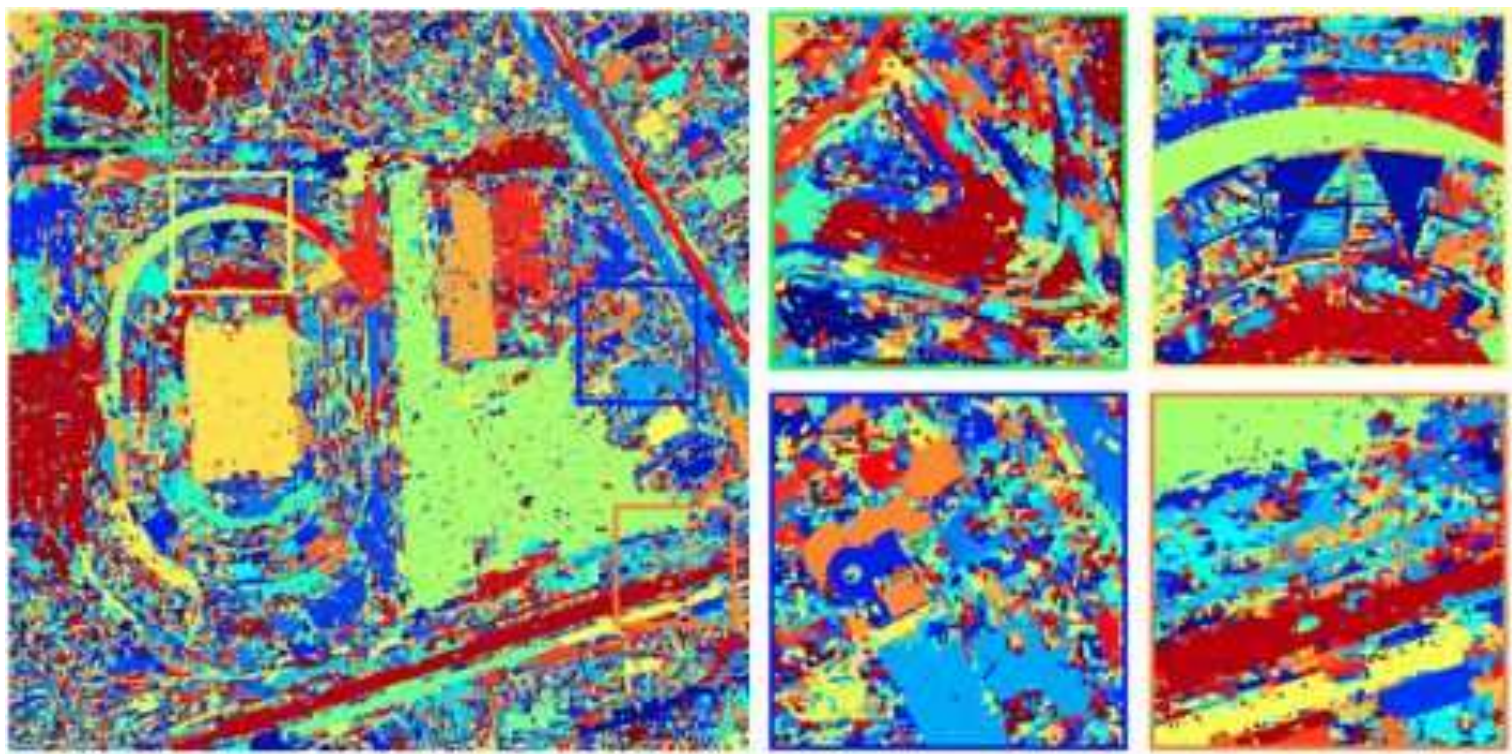

(b) $\mathrm{CC}_{\alpha}(\tau(\mathbf{I}))$ from $15(\mathrm{~b})$. \# CC $=58,954$
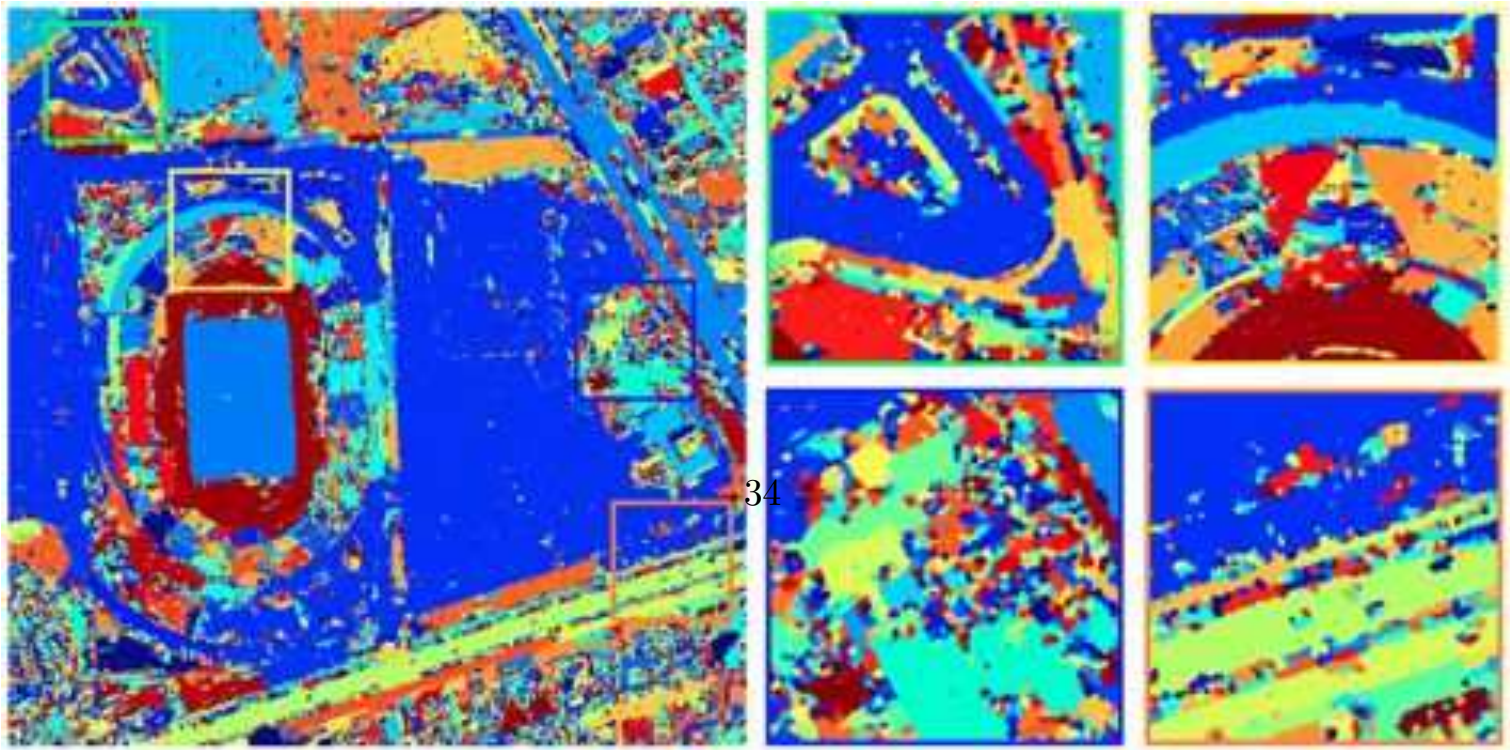

(c) $\mathrm{CC}_{\alpha}(\tilde{\tau}(\mathbf{I}))$ from $15(\mathrm{c}) . \# \mathrm{CC}=22,461$ 

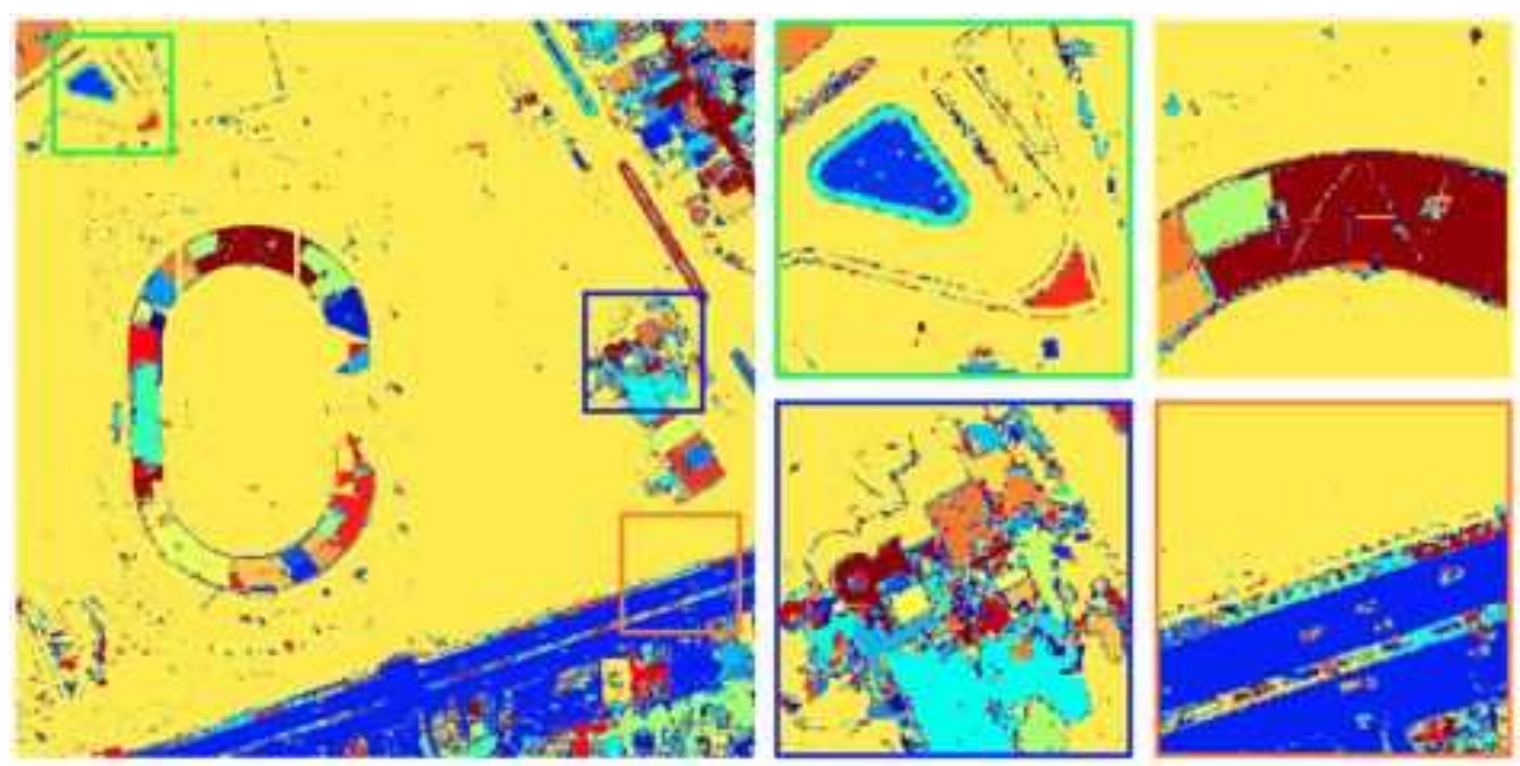

(a) $\mathrm{CC}_{\alpha}(\mathbf{I})$ from 15 (a). \# CC $=53,506$
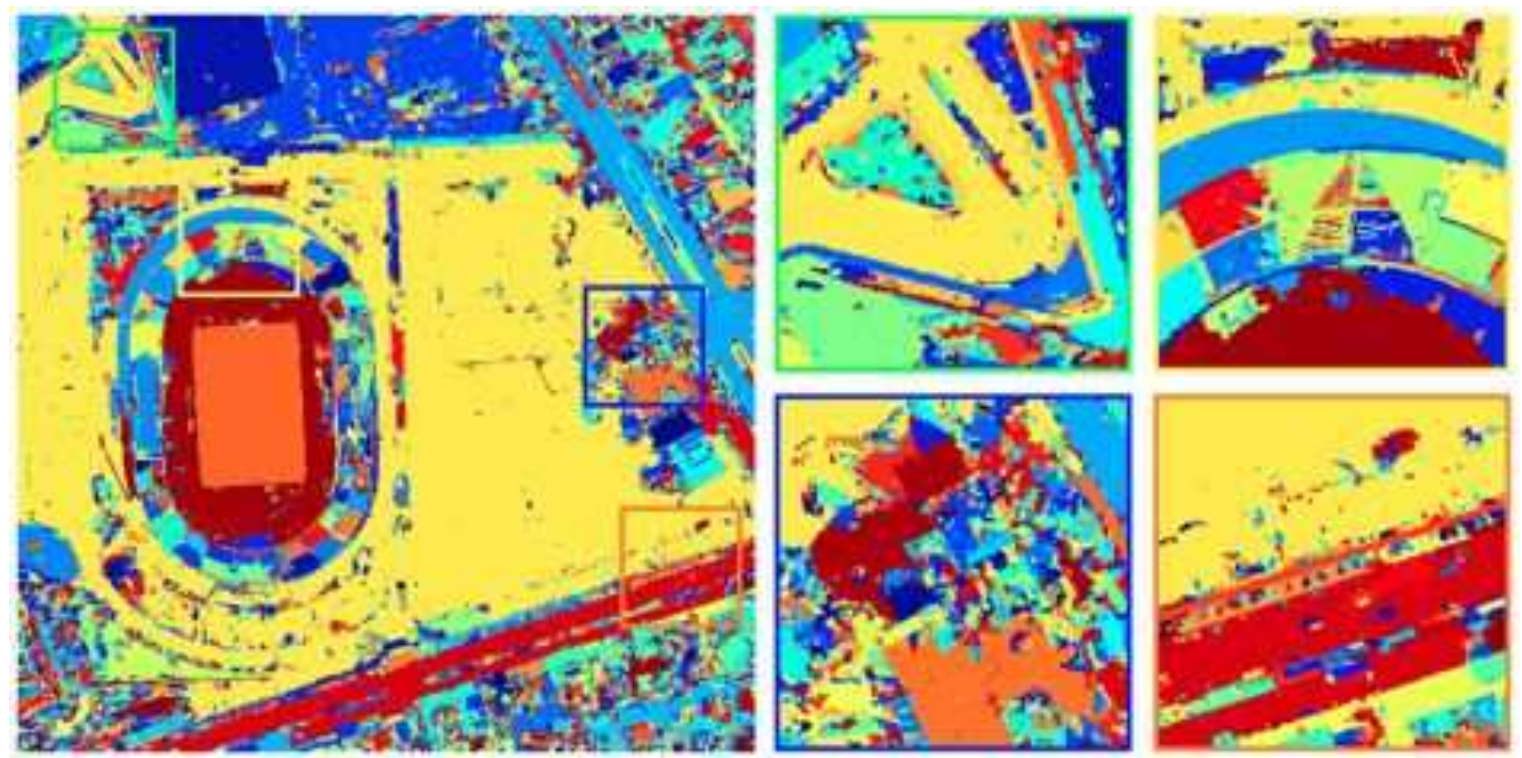

(b) $\mathrm{CC}_{\alpha}(\tau(\mathbf{I}))$ from $15(\mathrm{~b})$. \# CC $=24,234$
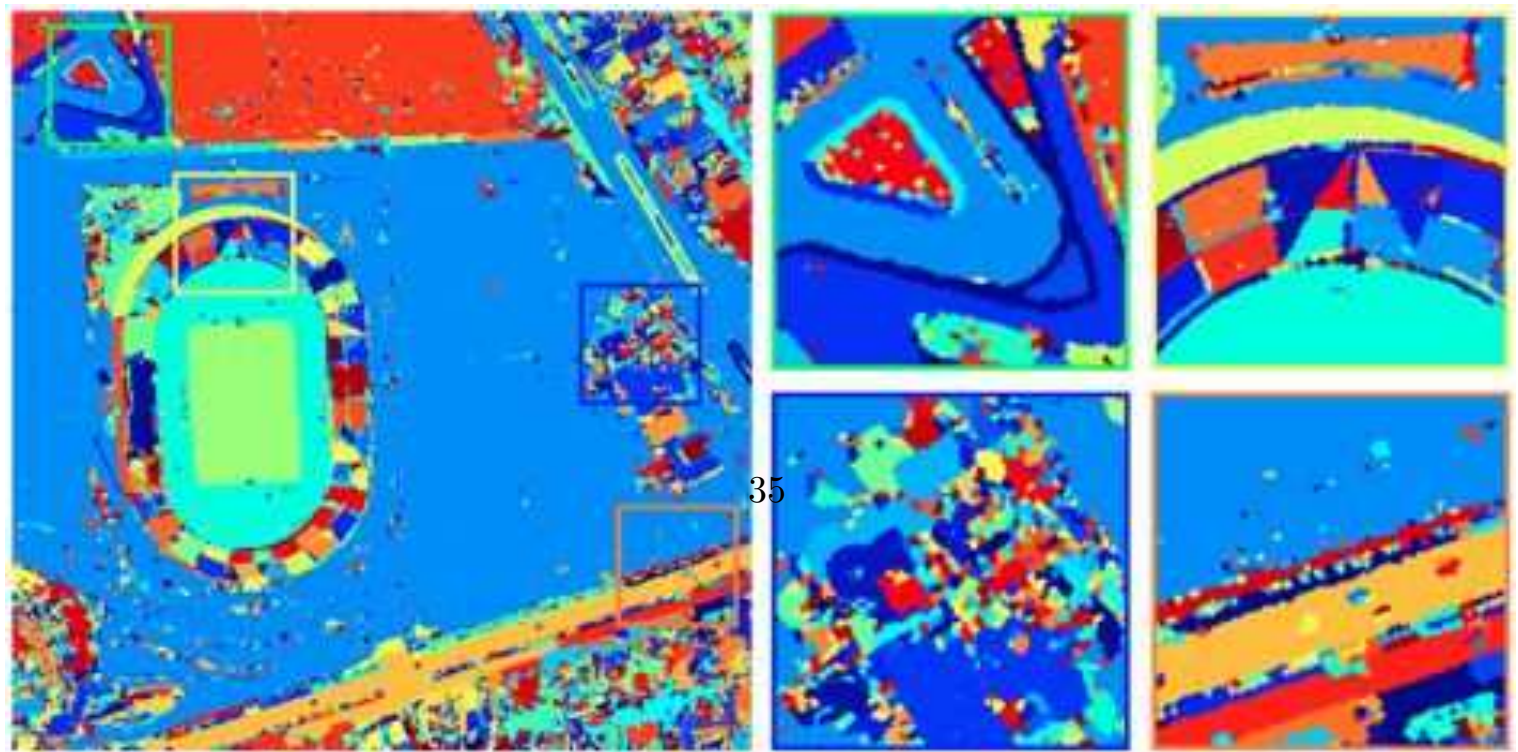

(c) $\mathrm{CC}_{\alpha}(\tilde{\tau}(\mathbf{I}))$ from $15(\mathrm{c}) . \# \mathrm{CC}=8,195$ 


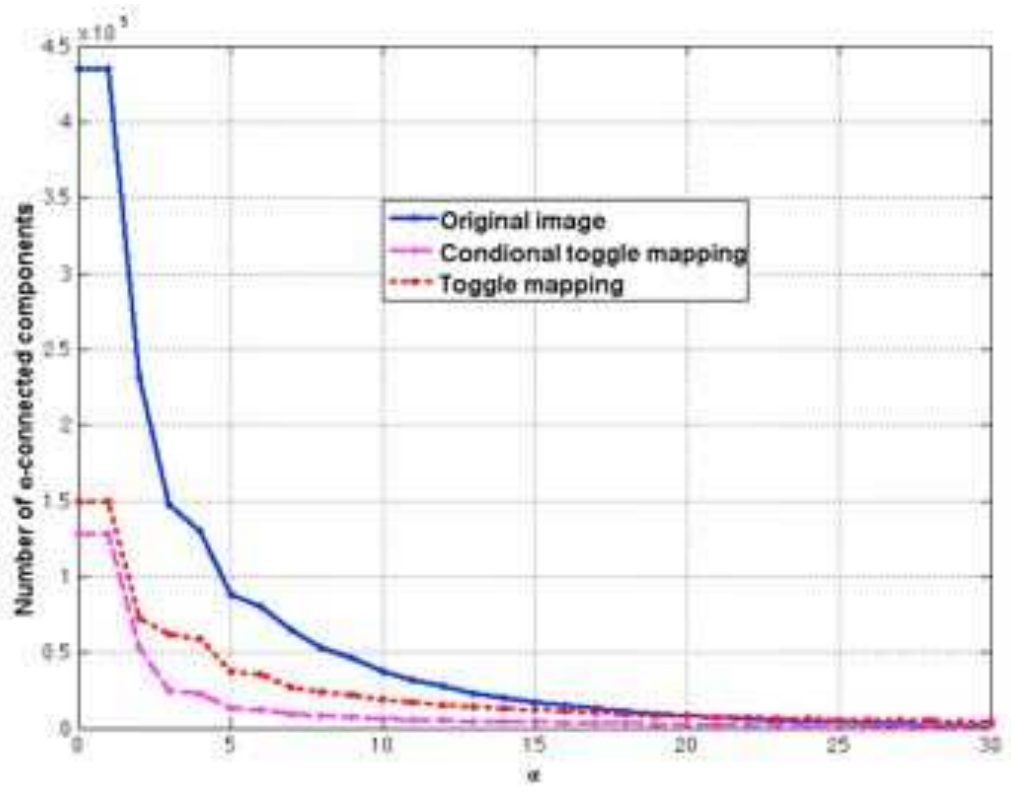

(a) $\alpha$ vs $\# \mathrm{CC}_{\alpha}$

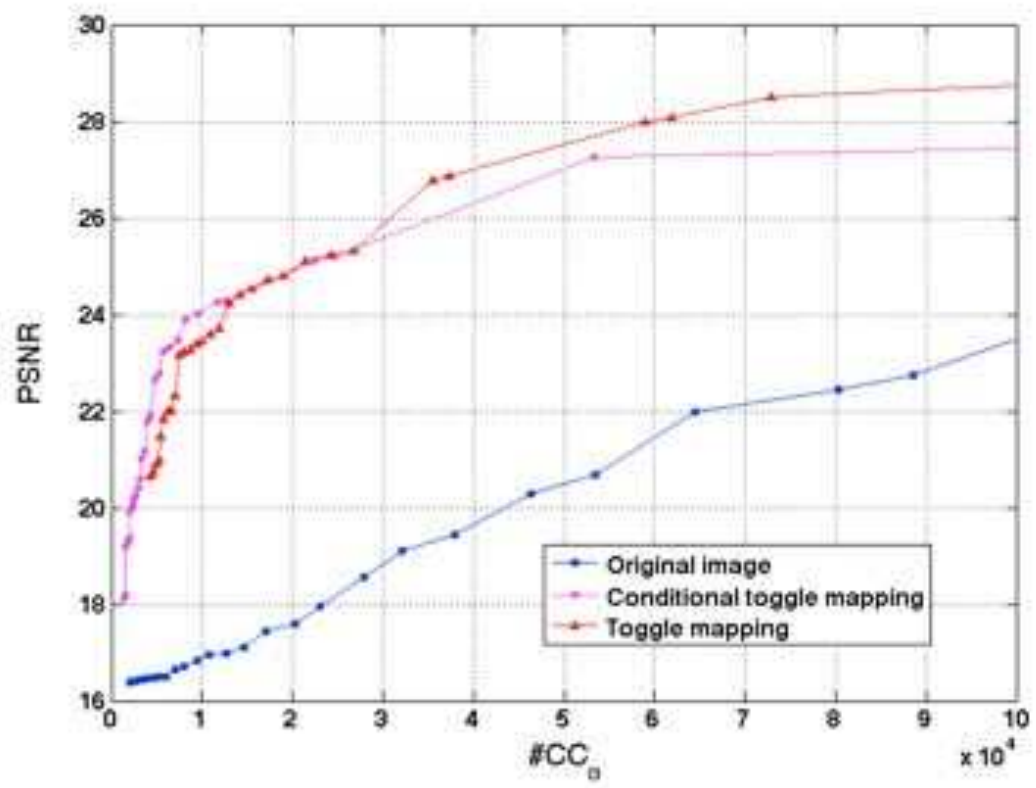

(b) $\# \mathrm{CC}_{\alpha}$ vs PSNR

Figure 18: (a) Illustrates the number connected components in the hierarchy induced by the parameter $\alpha$. Note than on the original image, $\mathrm{CC}_{\alpha}$ produces too many regions when $\alpha$ is small. That annoying effect is corrected by both classical and conditional toggle mapping. (b) PSNR of restored image by using the mean value per $\alpha$-connected component is presented for $\alpha=[0,1, \ldots, 30]$. 